\title{
Membrane Resonance in Pyramidal and GABAergic Neurons of the Mouse Perirhinal Cortex
}

\section{OPEN ACCESS}

Edited by:

Sonia Gasparini,

Louisiana State University,

United States

Reviewed by:

Rishikesh Narayanan, Indian Institute of Science (IISC), India

Rory McQuiston,

Virginia Commonwealth University,

United States

*Correspondence:

Francesca Talpo

francesca.talpo01@universitadipavia.it Gerardo Rosario Biella

gerardo.biella@unipv.it

${ }^{\dagger}$ These authors share first authorship

¥These authors have contributed equally to this work

Specialty section:

This article was submitted to

Cellular Neurophysiology,

a section of the journal

Frontiers in Cellular Neuroscience

Received: 30 April 2021

Accepted: 16 June 2021

Published: 22 July 2021

Citation:

Binini N, Talpo F, Spaiardi P, Maniezzi C, Pedrazzoli M, Raffin F, Mattiello N, Castagno AN, Masetto $S$, Yanagawa Y, Dickson CT, Ramat $S$,

Toselli M and Biella GR (2021)

Membrane Resonance in Pyramidal and GABAergic Neurons of the

Mouse Perirhinal Cortex.

Front. Cell. Neurosci. 15:703407.

doi: 10.3389/fncel.2021.703407

\begin{abstract}
Noemi Binini ${ }^{1 \dagger}$, Francesca Talpo ${ }^{1 * \dagger}$, Paolo Spaiardi ${ }^{1 \neq}$, Claudia Maniezzi ${ }^{1 \neq}$, Matteo Pedrazzoli', Francesca Raffin ${ }^{1}$, Niccolò Mattiello' ${ }^{1}$, Antonio N. Castagno', Sergio Masetto ${ }^{2}$, Yuchio Yanagawa ${ }^{3}$, Clayton T. Dickson ${ }^{4}$, Stefano Ramat ${ }^{5}$, Mauro Toselli ${ }^{1}$ and Gerardo Rosario Biella ${ }^{1 *}$

1 Department of Biology and Biotechnology Lazzaro Spallanzani, University of Pavia, Pavia, Italy, ${ }^{2}$ Department of Brain and Behavioral Sciences, University of Pavia, Pavia, Italy, ${ }^{3}$ Department of Genetic and Behavioral Neuroscience, Gunma University, Maebashi, Japan, ${ }^{4}$ Department of Psychology, University of Alberta, Edmonton, AB, Canada, ${ }^{5}$ Department of Industrial and Information Engineering, University of Pavia, Pavia, Italy
\end{abstract}

The perirhinal cortex (PRC) is a polymodal associative region of the temporal lobe that works as a gateway between cortical areas and hippocampus. In recent years, an increasing interest arose in the role played by the PRC in learning and memory processes, such as object recognition memory, in contrast with certain forms of hippocampus-dependent spatial and episodic memory. The integrative properties of the PRC should provide all necessary resources to select and enhance the information to be propagated to and from the hippocampus. Among these properties, we explore in this paper the ability of the PRC neurons to amplify the output voltage to current input at selected frequencies, known as membrane resonance. Within cerebral circuits the resonance of a neuron operates as a filter toward inputs signals at certain frequencies to coordinate network activity in the brain by affecting the rate of neuronal firing and the precision of spike timing. Furthermore, the ability of the PRC neurons to resonate could have a fundamental role in generating subthreshold oscillations and in the selection of cortical inputs directed to the hippocampus. Here, performing whole-cell patch-clamp recordings from perirhinal pyramidal neurons and GABAergic interneurons of GAD67$\mathrm{GFP}^{+}$mice, we found, for the first time, that the majority of PRC neurons are resonant at their resting potential, with a resonance frequency of $0.5-1.5 \mathrm{~Hz}$ at $23^{\circ} \mathrm{C}$ and of $1.5-2.8 \mathrm{~Hz}$ at $36^{\circ} \mathrm{C}$. In the presence of ZD7288 (blocker of HCN channels) resonance was abolished in both pyramidal neurons and interneurons, suggesting that $\mathrm{l}_{\mathrm{h}}$ current is critically involved in resonance generation. Otherwise, application of $\Pi x$ (voltagedependent $\mathrm{Na}^{+}$channel blocker) attenuates the resonance in pyramidal neurons but not in interneurons, suggesting that only in pyramidal neurons the persistent sodium current has an amplifying effect. These experimental results have also been confirmed by a computational model. From a functional point of view, the resonance in the PRC would affect the reverberating activity between neocortex and hippocampus, especially during slow wave sleep, and could be involved in the redistribution and strengthening of memory representation in cortical regions.

Keywords: membrane resonance, pyramidal neuron, GABAergic interneuron, perirhinal cortex, patch-clamp 


\section{INTRODUCTION}

The perirhinal cortex (PRC) is a polymodal associative ventral region of the temporal lobe located laterally to the rhinal sulcus. It is connected with many sensory and polymodal areas, reward-related cortices, and other structures of the medial temporal lobe (MTL) such as the entorhinal and postrhinal (or parahippocampal in primates) cortices, the amygdala (Pinto et al., 2006; Biella et al., 2010), and the hippocampus (Witter et al., 2000; Kealy and Commins, 2011; Suzuki and Naya, 2014). In recent years, there has been an increasing interest in the role played by the perirhinal cortex in cognitive functions such as declarative learning and memory, as well as interest in its susceptibility during the initial stages of specific neurodegenerative diseases like Alzheimer's. More specifically, PRC is involved in recognition memory, visual perception, and associative processes. Our current understanding of the role of PRC has been primarily based on lesion experiments, but the underlying functional mechanisms determining how it executes these tasks remain to be uncovered. Based on cytoarchitectonic properties and anatomical connections, PRC should play a crucial role in processing information directed from and to the hippocampal formation. The key role of the perirhinal region, that is not simply a passive relay station, is underscored. Indeed, PRC has a robust inhibitory system (de Curtis and Paré, 2004) that acts as an active gate to selectively allow information to propagate from other cortical regions to the hippocampus and vice versa. In the PRC, in fact, excitatory neocortical inputs undergo a powerful inhibitory block maintained by local GABAergic interneurons (Martina et al., 2001; Biella et al., 2002; Garden et al., 2002; Willems et al., 2018). Moreover, PRC importantly retains integrative properties that are essential for different memory and perceptual tasks (Biella et al., 2001; Murray and Richmond, 2001; Davachi, 2006; Staresina and Davachi, 2008; Suzuki and Naya, 2014).

According to this state of the art, we suggest that the inhibitory barrier in the PRC could allow a selection of relevant inputs directed to and from the hippocampus. To this purpose, namely the ability to select and enhance specific inputs, different cellular and network strategies could be applied, like for example to enhance synaptic plasticity (Perugini et al., 2012) as well as to generate oscillatory patterns in neuronal networks. Furthermore, it has been shown that subpopulations of neurons in many cerebral areas, such as neocortex (Hutcheon et al., 1996, Sun et al., 2014), entorhinal cortex (Lampl and Yarom, 1997; Hutcheon and Yarom, 2000; Haas and White, 2002; Erchova et al., 2004; Engel et al., 2008), and hippocampus (Leung and Yu, 1998; Pike et al., 2000; Hu et al., 2002; Erchova et al., 2004; Narayanan and Johnston, 2007), are able to amplify the output voltage to current input at selected frequencies. This property is called membrane resonance. Within cerebral circuits the resonance of different types of neurons operates as a filter toward inputs signals at certain frequencies, to coordinate network activity in the brain by affecting the rate of neuronal firing (Hutcheon and Yarom, 2000), to influence the precision of spike timing (Desmaisons et al., 1999; Haas and White, 2002; Schaefer et al., 2006), and to cause spike clustering (Chen and Shepherd, 1997; Desmaisons et al., 1999;
Pedroarena et al., 1999; Wu et al., 2001; Izhikevich et al., 2003). Resonant behavior emerges as a result of an interplay between membrane passive and active properties due to the frequencydependent increase of membrane impedance produced by voltage-dependent ion channels (Hutcheon and Yarom, 2000). Several voltage-dependent ion currents are critical for the genesis of the resonance such as M-type potassium current, T-type calcium current, h-type hyperpolarized-activated cationic current, whereas other ones such as the persistent sodium current or the potassium inward rectifier current can act as amplifying currents that facilitate membrane resonance. The ability of perirhinal neurons to resonate could have a fundamental role in generating subthreshold oscillations and in the selection of cortical inputs directed to the hippocampus. At present, there is no report about a possible resonant behavior of neurons located in the PRC.

Shay et al. (2012) demonstrated that, among the different areas of the parahippocampal region, the neurons of the medial entorhinal cortex (EC) show a maximum resonant frequency ranging from 4 to $8 \mathrm{~Hz}$ (theta wave) suggesting that these properties could contribute to the generation of the firing dynamics of grid cells in the medial EC that are related to the processing of spatial information. Neurons recorded from the lateral EC that is strongly connected to the PRC (Burke et al., 2018) showed a lower resonant frequency, between 1 and $2 \mathrm{~Hz}$, very similar to that observed in neocortical neurons (0.7$2.5 \mathrm{~Hz}$ ) (Hutcheon et al., 1996). Furthermore, resonant behavior has been observed in different subregions of the hippocampus. Hippocampal pyramidal neurons showed a resonant frequency ranging among $2-8 \mathrm{~Hz}$ (Pike et al., 2000; Hu et al., 2002) whereas two subpopulations of GABAergic interneurons showed either a lower (1-3 Hz, the horizontal interneuron) or higher (10$50 \mathrm{~Hz}$, fast-spiking interneurons) resonant frequency bandwidth. Likely, the resonance properties of neurons and their synaptic interactions within the different areas of the hippocampal region underlie state-dependent network oscillations that may serve as a temporal modality to associate linked and distant neural networks. In this way, by binding different neural networks, resonance could act as a potential cellular mechanism to temporally coordinate information directed to and from the hippocampus.

A systematic study of the resonance properties in the perirhinal neurons has not been performed yet. In this study, we report for the first time that a sizeable subpopulation of both pyramidal cells and GABAergic interneurons of the PRC are able to resonate in a selected range of frequencies $(1-2.5 \mathrm{~Hz})$.

\section{MATERIALS AND METHODS}

\section{Animals and Brain Slice Preparation}

Juvenile (P17-P27) heterozygous GAD67-GFP knock-in mice (Tamamaki et al., 2003) were used for all experiments. Experimental handling of the animals was performed in accordance with EU directive $86 / 609 / \mathrm{EEC}$, approved by the National Ministry of Health, and designed to minimize the number of the animals and their suffering. 
Animals were anesthetized by inhalation of isoflurane and decapitated. The whole brain was removed and submerged in cold $\left(\sim 4^{\circ} \mathrm{C}\right)$ carboxygenated $\left(95 \% \mathrm{O}_{2}, 5 \% \mathrm{CO}_{2}\right)$ cutting solution (Sucrose $70 \mathrm{mM}, \mathrm{NaCl} 80 \mathrm{mM}, \mathrm{KCl} 2.5 \mathrm{mM}, \mathrm{NaHCO}_{3} 26 \mathrm{mM}$, Glucose $15 \mathrm{mM}, \mathrm{MgCl}_{2} 7 \mathrm{mM}, \mathrm{CaCl}_{2} 1 \mathrm{mM}, \mathrm{NaH}_{2} \mathrm{PO}_{4} 1.25 \mathrm{mM}$; $\mathrm{pH}$ 7.3). Coronal $350 \mu \mathrm{m}$-thick slices containing the rhinal sulcus were prepared using a vibratome (DTK-1000, Dosaka EM). Following cutting, the slices were allowed to equilibrate for at least $1 \mathrm{~h}$ in a recovery chamber filled with carboxygenated artificial cerebrospinal fluid (aCSF) medium ( $\mathrm{NaCl} 125 \mathrm{mM}, \mathrm{KCl}$ $2.5 \mathrm{mM}, \mathrm{NaHCO}_{3} 26 \mathrm{mM}$, Glucose $15 \mathrm{mM}, \mathrm{MgCl}_{2} 1.3 \mathrm{mM}$, $\mathrm{CaCl}_{2} 2.3 \mathrm{mM}, \mathrm{NaH}_{2} \mathrm{PO}_{4} 1.25 \mathrm{mM}$; pH 7.3).

\section{Electrophysiological Recordings}

Recordings were performed at room temperature $\left(\sim 23^{\circ} \mathrm{C}\right)$ on submerged slices perfused at $1.4 \mathrm{ml} / \mathrm{min}$ with aCSF, unless otherwise stated. The recording chamber was mounted on an E600FN microscope connected to a near-infrared CCD camera. Data were derived from perirhinal pyramidal neurons and GAD67-GFP-expressing GABAergic interneurons using the whole-cell patch-clamp technique in voltage- and current-clamp modes. Pipettes were produced from borosilicate glass capillary tubes (Hilgenberg GmbH) using a horizontal puller (P-97, Sutter instruments) and filled with the following intracellular solution: K-gluconate $130 \mathrm{mM}, \mathrm{NaCl} 4 \mathrm{mM}, \mathrm{MgCl}_{2} 2 \mathrm{mM}$, EGTA $1 \mathrm{mM}$, creatine phosphate $5 \mathrm{mM}, \mathrm{Na}_{2}$ ATP $2 \mathrm{mM}, \mathrm{Na}_{3} \mathrm{GTP}$ $0.3 \mathrm{mM}$, Hepes $10 \mathrm{mM}$ ( $\mathrm{pH} 7.3$ with $\mathrm{KOH}$ ). Series resistance was minimized and monitored throughout the experiment $\left(R_{S}\right.$ initial $=9.2 \pm 0.3 ; R_{s}$ final $=11.7 \pm 0.5$ for pyramidal neurons, $N=128$ and $\mathrm{R}_{\mathrm{s}}$ initial $=11.7 \pm 0.5 ; \mathrm{R}_{\mathrm{s}}$ final $=13.8 \pm 0.5$ for GABAergic interneurons, $N=70$ ), however, it was not compensated by using the bridge balance circuit. Recordings were made with a MultiClamp 700B amplifier (Molecular Devices) and digitized with a Digidata 1322 computer interface (Molecular Devices). Data were acquired using the software Clampex 9.2 (Molecular Devices), sampled at $20 \mathrm{kHz}$, filtered at $10 \mathrm{kHz}$, and analyzed with the software Clampfit 10.2 (Molecular Devices) and Origin 6.0 (Microcal).

\section{Morphology of Recorded Neurons}

To confirm the identity of the recorded cells by their morphology, biocytin ( $3 \mathrm{mg} / \mathrm{ml}$, Sigma) was added to the intracellular solution and it diffused into the cell through the patch micropipette during electrophysiological recording. Following recordings slices were fixed in $4 \%$ paraformaldehyde for $30 \mathrm{~min}$, washed with PBS (Dulbecco's phosphate buffer saline, Sigma), then incubated overnight with $10 \mu \mathrm{g} / \mathrm{ml}$ DAPI (4',6-diamidino-2-phenylindole), and $5 \mu \mathrm{g} / \mathrm{ml}$ Alexa Fluor 568-conjugated streptavidin (Molecular Probes). Next day slices were mounted on microscope slides using DAKO Mounting Medium. Images were acquired by confocal microscopy [microscope Leica TCS SP2 equipped with three laser lines: (i) Ar/UV laser with emissions at 351 and $364 \mathrm{~nm}$; (ii) Ar/Vis laser with emissions at 458 and $488 \mathrm{~nm}$ and (iii) HeNe laser with emissions at 543 and $633 \mathrm{~nm}$ ] using a dedicated acquisition software (LCS software). Cells were reconstructed by using Imaris software (Bitplane).

\section{Characterization of the Passive and the Firing Properties of the Neurons}

For each recorded cell we calculated the membrane capacitance $\left(C_{m}\right)$, the input resistance $\left(R_{i n}\right)$, and the resting membrane potential $\left(\mathrm{V}_{\mathrm{r}}\right) \cdot \mathrm{C}_{\mathrm{m}}$ was estimated by integrating the capacitive current evoked by a $-10 \mathrm{mV}$ pulse, whereas $R_{\text {in }}$ was calculated from the same protocol at the end of a $20 \mathrm{~ms}$ pulse, when the current trace reached the steady state. $\mathrm{V}_{\mathrm{r}}$ was detected in current-clamp mode with $0 \mathrm{pA}$ current injection. To characterize the features of the action potentials (APs), we injected positive supra-threshold current steps. The AP threshold $\left(\mathrm{AP}_{\mathrm{Th}}\right)$ is the value of the membrane potential at which a rapid upstroke of the AP starts (corresponding to the value of potential at which the action potential temporal derivative crosses $20 \mathrm{~V} / \mathrm{s}$ ). The AP amplitude $\left(\mathrm{AP}_{\mathrm{A}}\right)$ was measured as the voltage difference between the top of the spike and the $\mathrm{AP}_{\mathrm{Th}}$. The AP duration $\left(\mathrm{AP}_{\mathrm{D}}\right)$ was calculated as the spike width measured at half-maximal spike amplitude. The firing pattern of each cell was categorized through a twofold quantitative analysis. Specifically, we evaluated i) the inter-spike-intervals (ISIs) and ii) the coefficients of variation for a sequence of ISIs (CV2) (Holt et al., 1996; Shinomoto et al., 2009; Komendantov et al., 2019; Graf et al., 2020). ISIs were computed as the temporal separation of two contiguous spikes repeated for each pair of APs of the neuronal discharge. CV2 was calculated as in Holt et al. (1996) by the following Equation (1) applied for all the ISIs of the discharge:

$$
\mathrm{CV} 2=\frac{2 *\left|\mathrm{ISI}_{\mathrm{i}+1}-\mathrm{ISI}_{\mathrm{i}}\right|}{\mathrm{ISI}_{\mathrm{i}+1}+\mathrm{ISI}_{\mathrm{i}}}
$$

\section{Characterization of Subthreshold Resonance}

To characterize the resonant behavior of the cells, the impedance amplitude profile (ZAP) method was used (Hutcheon et al., 1996; Hutcheon and Yarom, 2000). A sinusoidal current with constant amplitude (60 pA peak-to-peak) and linearly increasing frequency from 0 to $15 \mathrm{~Hz}$ (ZAP current of $50 \mathrm{~s}$ ) was applied. The protocol was repeated 3-5 times for each cell and the voltage responses were recorded and averaged. Resonance occurs as a peak in the voltage response at a specific frequency $\left(\mathrm{F}_{\mathrm{res}}\right)$. In some experiments the reproducibility of the voltage response has been demonstrated by applying modified ZAP current protocols (i.e., ZAP current with a 40 pA or a 20-pA peak-to-peak amplitude, inverted ZAP current with a $15-0 \mathrm{~Hz}$ declining frequency, negative ZAP current with the first peak of the stimulus oriented negatively). The impedance profile $[\mathrm{Z}(\mathrm{f})]$ was calculated by dividing the Fast Fourier Transform (FFT) of the membrane potential response (V) by the FFT of the ZAP current (I), as indicated in Equation (2).

$$
Z(f)=\frac{F F T[V(t)]}{F F T[I(t)]}
$$

$\mathrm{Z}(\mathrm{f})$ is a complex quantity $(\mathrm{Z}(\mathrm{f})=\mathrm{Z}$ Real+ iZImaginary) that can be plotted as a vector whose magnitude $(|\mathrm{Z}(\mathrm{f})|)$ 
and phase $\left(\varphi_{\mathrm{z}}(\mathrm{f})\right)$ are given by the expressions (3) and (4), respectively.

$$
\begin{gathered}
|Z(f)|=\sqrt{(Z, \text { Real })^{2}+(Z, \text { Imaginary })^{2}} \\
\phi_{z}(f)=\tan ^{-1}\left(\frac{Z, \text { Imaginary }}{Z, \text { Real }}\right)
\end{gathered}
$$

The plot of the impedance phase as a function of frequency indicates the phase shift of the voltage wave relative to the current wave. Throughout this manuscript the term impedance refers to the magnitude of the impedance vector, unless otherwise stated. In the relationship between impedance and frequency, the ratio of the impedance at the resonance peak $\left(Z_{\text {res }}\right)$ to the impedance at $0.1 \mathrm{~Hz}\left(\mathrm{Z}_{0}\right)$ is called $Q$-value and this parameter is used to highlight the absence $(Q<1.05)$ or the presence $(Q \geq 1.05)$ of resonance and its relative strength. The complex representation of the impedance integrates the information of both magnitude and phase. The impedance magnitude corresponds to the length of the vectors connecting the origin of the axes to each point of the graph, while the phase is the angle between each vector and the real axis (see Figure 2D). For resonant cells, the complex representation is characterized by points in both positive and negative regions of the imaginary axes whereas non-resonant neurons display a series of points limited to the negative imaginary region.

\section{Chemicals and Drugs}

All drugs were added to the aCFS medium and bath perfused at the following final concentrations: $10 \mu \mathrm{M}$ 4-Ethylphenylamino1,2-dimethyl-6-methylaminopyrimidin chloride (ZD7288, Abcam; HCN channel blocker), $1 \mu \mathrm{M}$ tetrodotoxin (TTx, Alomone Labs; voltage-dependent $\mathrm{Na}^{+}$channel blocker), 10 $\mu \mathrm{M}$ 2,3-Dioxo-6-nitro-1,2,3,4-tetrahydrobenzo[f] quinoxaline7-sulfonamide (NBQX, Tocris; AMPA receptors antagonist), $30 \mu \mathrm{M}$ (RS)-3-(2-Carboxypiperazin-4-yl)-propyl-1-phosphonic acid [(RS)-CPP, Tocris; NMDA receptors antagonist] and $10 \mu \mathrm{M}$ bicuculline methiodide (Sigma-Aldrich; $\mathrm{GABA}_{\mathrm{A}}$ receptors antagonist).

\section{Computational Model}

A conductance-based single compartment model reproducing subthreshold resonance was developed using MATLAB. It includes a passive leak current $\left(\mathrm{I}_{\text {leak }}\right.$ ), a hyperpolarizationactivated cation current $\left(\mathrm{I}_{\mathrm{h}}\right)$, and a persistent (non-inactivating) $\mathrm{Na}^{+}$current $\left(\mathrm{I}_{\mathrm{NaP}}\right)$. In the model, these currents were described using the following equations:

$$
\begin{gathered}
\mathrm{I}_{\text {leak }}=\mathrm{g}_{\text {leak }} \times\left(\mathrm{V}-\mathrm{E}_{\text {leak }}\right) \\
\mathrm{I}_{\mathrm{h}}=\mathrm{g}_{\mathrm{h}} \times \mathrm{f} \times\left(\mathrm{V}-\mathrm{E}_{\mathrm{h}}\right) \\
\mathrm{I}_{\mathrm{Nap}}=\mathrm{g}_{\mathrm{Nap}} \times \mathrm{w} \times\left(\mathrm{V}-\mathrm{E}_{\mathrm{Nap}}\right)
\end{gathered}
$$

with $g_{\text {leak, }} \mathrm{g}_{\mathrm{h}}$, and $\mathrm{g}_{\mathrm{NaP}}$ being the maximal conductances of the corresponding currents and $\mathrm{E}_{\text {leak }}, \mathrm{E}_{\mathrm{h}}$, and $\mathrm{E}_{\mathrm{NaP}}$ their reversal potentials.
Moreover, the dynamics of the state variables $x_{i}=f$ were described by the following equation:

$$
\frac{\mathrm{dx}_{\mathrm{n}}}{\mathrm{dt}}=\frac{\mathrm{x}_{\mathrm{n} \infty}(\mathrm{V})-\mathrm{x}_{\mathrm{n}}}{\tau_{\mathrm{xn}}}
$$

where $x_{n} \infty$ were the steady-state values of $x_{n}$ and $\tau_{x n}$ were the corresponding time constants.

A summary of the reversal potentials and the equations that define the steady-state variables and time constants for the different currents is shown in Table 1. Voltage dependence of state variables and time constants for $\mathrm{I}_{\mathrm{h}}$ and for $\mathrm{I}_{\mathrm{NaP}}$ were taken from Spain et al. (1987) and Hodgkin and Huxley (1952), respectively.

The $\tau$ value for $I_{h}$ was divided by the temperature-correcting factor (Magee, 1998):

$$
4.5^{(\mathrm{T}-38) / 10}
$$

where $\mathrm{T}$ is the temperature in degree (Celsius).

The model reproduces the stimulus current used in the experiments $\left(\mathrm{I}_{\mathrm{Zap}}\right)$. A holding current $\left(\mathrm{I}_{\mathrm{cmd}}\right)$ was also added to the model to maintain a holding potential of $-70 \mathrm{mV}$.

The output of the model is the variation of voltage vs. time, which is represented by the following equation:

$$
\frac{\mathrm{dV}}{\mathrm{dt}}=\frac{\mathrm{I}_{\text {Zap }}-\mathrm{I}_{\text {leak }}-\mathrm{I}_{\mathrm{h}}-\mathrm{I}_{\mathrm{Zap}}+\mathrm{I}_{\mathrm{Cmd}}}{\mathrm{C}}
$$

in which $\mathrm{C}$ is the membrane capacitance, fixed at $1 \mu \mathrm{F} / \mathrm{cm}^{2}$.

\section{Statistics}

Collected data are presented as all-point plots together with summary statistics [means \pm standard error of the mean (SEM.)] to show them in detail and avoid misinterpretations (Marder and Taylor, 2011; Rathour and Narayanan, 2019). Statistical significance was determined, depending on data, by paired or unpaired two-tailed Student's $t$-test, One-Way ANOVA test followed by Bonferroni post-hoc, or KruskalWallis test with Dunn's multiple comparisons test. To assess pairwise relationship in the parameters under investigation we analyzed the scatterplot matrices of them and computed the correspondent Pearson's correlation coefficients and significance values, as in Mishra and Narayanan (2020).

TABLE 1 | Parameters and equations used for calculation of ionic currents in the computational model.

\begin{tabular}{lccccc}
\hline Current & $\mathbf{g}\left(\mathbf{m s} / \mathbf{c m}^{2}\right)$ & $\mathbf{V}_{\text {rev1 }}$ & $\mathbf{V}_{\text {rev2 }}$ & State variables & $\tau$ (ms) \\
\hline$l_{\text {leak }}$ & 0.03 & -89 & -89 & - & - \\
$l_{h}$ & 0.009 & -25 & -25 & $f_{\infty}=\frac{1}{1+e^{(V+70)} / 7}$ & 38 \\
$\mathrm{I}_{\mathrm{NaP}}$ & 0.23 & 70.6 & 74.2 & $w_{\infty}=\frac{1}{1+e^{-(V+70)} / 5}$ & 5 \\
\hline
\end{tabular}

Summary of conductance values (g), reversal potentials, equations ruling steadystate variables, and time constants $(\tau)$ for ionic currents implemented in the model. Voltage dependence of state variables and time constants were taken from Spain et al. (1987) for $\mathrm{I}_{\mathrm{h}}$ and from Hodgkin and Huxley (1952) for $\mathrm{I}_{\mathrm{NaP}}$. 


\section{RESULTS}

\section{Properties and Firing Patterns of Pyramidal Neurons and GABAergic Interneurons of the Mouse PRC}

Targeted whole-cell patch-clamp recordings were performed on both deep and superficial layer pyramidal cells and GABAergic interneurons from coronal PRC (areas 35 and 36) brain slices obtained from young GAD67-GFP knock-in mice. In this animal model GABAergic interneurons can be easily identified because they are constitutively labeled with GFP (Tamamaki et al., 2003). In a subset of cells, confirmation of anatomical identity was obtained by biocytin labeling and imaging (Figures 1A,F). From a functional point of view, we found significant differences in passive membrane properties between pyramidal cells and GABAergic interneurons, as expected (Karayannis et al., 2007). Specifically, membrane capacitance $\left(C_{m}\right)$ was significantly higher in pyramidal neurons than in GABAergic interneurons $(p<0.001)$, while membrane input resistance $\left(\mathrm{R}_{\mathrm{in}}\right)$ was significantly higher in GABAergic interneurons as compared with pyramidal neurons ( $p<0.01$ ) (Table 2 ). Instead, we found no differences in resting membrane potential $\left(\mathrm{V}_{\mathrm{r}}\right)$ (Table 2). Then, we analyzed the firing patterns of the PRC pyramidal neurons and GABAergic interneurons by calculating (i) the distribution of the inter-spike-intervals (ISI) (Figure 1L) and (ii) the coefficient of variation of the discharge (CV2), that measures the intrinsic variability of a spike train (Figure 1M; Holt et al., 1996; Shinomoto et al., 2009; Komendantov et al., 2019; Graf et al., 2020).

In this way, we identified 5 different types of discharge (Figures 1B-E,G-K).

(1) Late-spiking regular neurons (RS): At just-suprathreshold, these neurons show a slow ramp depolarization before the onset of their spike trains, with a consequent delay of the first spike. At more sustained depolarizations, they are characterized by a persistent tonic or slightly adapting firing. In line with this, their ISI distribution is linear and almost parallel to the $X$-axis (red dots in Figure 1L). Also, their ISI-CV2 relationship shows a cloud of dots very concentrated and close to each other at a low CV2 (about 0.1) (red dots in Figure 1M).

(2) Stuttering fast-spiking neurons (FS): At justsuprathreshold, these neurons fire trains of high-frequency spikes $(30-50 \mathrm{~Hz})$ separated by variable periods of silence. At more sustained depolarizations, they are characterized by a persistent high-frequency $(50-100 \mathrm{~Hz})$ tonic firing. Likewise in RS neurons, their ISI distribution is linear and almost parallel to the $X$-axis and their ISI-CV2 relationship shows a cloud of dots very concentrated and close to each other at a low CV2 (about 0.1). However, the FS dots (orange) can be distinguished from the RS dots (red) because they are shifted to lower ISI values (Figures 1L,M).

(3) Adapting neurons (ADP): Adapting neurons typically begin their spike trains at a short latency following onset of a depolarizing current step and accommodate strongly. Due to adaptation, their ISI distribution is linear, but with a higher angular coefficient than RS and FS neurons (blue dots in Figure 1L). Also, their ISI-CV2 relationship shows a quite dispersed cloud of dots (blue dots in Figure 1M) with a higher mean CV2 (about 0.4).

(4) Bursting neurons (BST): Bursting neurons are characterized by spikes that occur in a stereotyped pattern consisting into a cluster of 2-3 action potentials riding on a slow depolarizing wave and followed by a strong slow afterhyperpolarization. After the burst, their firing generally becomes regular. Therefore, their ISI distribution is not linear but starts with shorter ISIs (green dots in Figure 1L) and their ISI-CV2 relationship consists in a rather compact cloud of dots (corresponding to the regular firing) accompanied by two or three more dispersed dots (corresponding to the burst) (green dots in Figure 1M).

(5) Irregular neurons (IR): Irregular neurons show a random and unpredictable firing pattern. Their ISI distribution is dispersed and not linear (black dots in Figure 1L) and also their ISI-CV2 relationship consists in a dispersed cloud of dots (black dots in Figure 1M). They have a mean CV2 similar to that of adapting neurons (about 0.4), but their non-linear ISI distribution uniquely characterizes them.

This classification is similar to what is currently described in literature (Faulkner and Brown, 1999; Beggs et al., 2000; McGann et al., 2001). Accordingly, we found that $41.5 \%$ of pyramidal neurons were RS, 20.5\% ADP, 26\% BST, and 12\% IR. The same classification indicates that $60 \%$ of GABAergic interneurons were FS, $18.5 \%$ RS, 3\% ADP, 10\% BST, and 8.5\% IR (Figure 1N).

\section{Resonant and Non-resonant Behavior in Glutamatergic Pyramidal Neurons and GABAergic Interneurons of the PRC}

A regular 50sec-long ZAP current input with linearly increasing frequency from 0 to $15 \mathrm{~Hz}$ was applied to test for resonant behavior of pyramidal neurons (Figures 2A,E) and GABAergic interneurons (Figures $\mathbf{2} \mathbf{A}^{\prime}, \mathbf{E}^{\prime}$ ) at a membrane potential of -70 $\mathrm{mV}$. Resonance appears as a peak in the voltage response at a specific frequency $\left(\mathrm{F}_{\text {res }}\right)$ (Figures $\left.\mathbf{2 A}, \mathbf{A}^{\prime}\right)$, that is absent in nonresonant cells (Figures $2 \mathrm{E}, \mathbf{E}^{\prime}$ ). As a consequence, resonant cells show a peak in the impedance-to-frequency relationship at $\mathrm{F}_{\text {res }}$ (corresponding to the dashed vertical line in Figures $\mathbf{2 B}, \mathbf{B}^{\prime}$ ), whereas a clear peak is not detectable in non-resonant cells (Figures 2F, $\mathbf{F}^{\prime}$ ). Accordingly, the phase shift-to-frequency relationship and the complex representation of the impedance differentiates between resonant (Figures $2 \mathbf{C}, \mathbf{D}, \mathbf{C}^{\prime}, \mathbf{D}^{\prime}$ ) and nonresonant (Figures $\mathbf{2} \mathbf{G}, \mathbf{H}, \mathbf{G}^{\prime}, \mathbf{H}^{\prime}$ ) neurons, through clustering of positive values in resonant neurons. The percentage of the resonant pyramidal neurons and GABAergic interneurons measured in the superficial and deep layers of areas 35 (A35) and 36 (A36) of the PRC is shown in Table 3. Overall, the majority of perirhinal pyramidal neurons (77\%) and GABAergic interneurons (54\%) were resonant and were equally distributed throughout the PRC, without a clear prevalence in a specific 


\section{Pyramidal neurons}

A

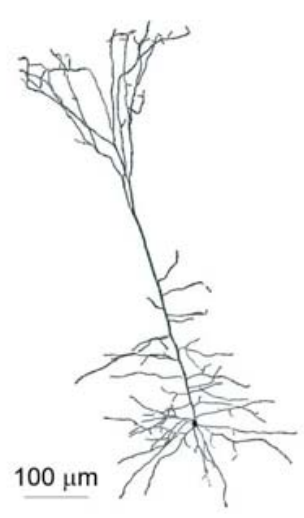

B

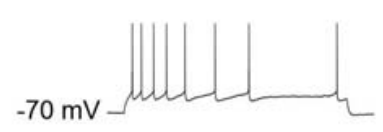

D

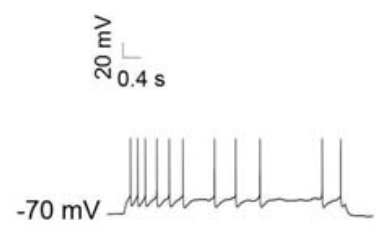

Interneurons

F

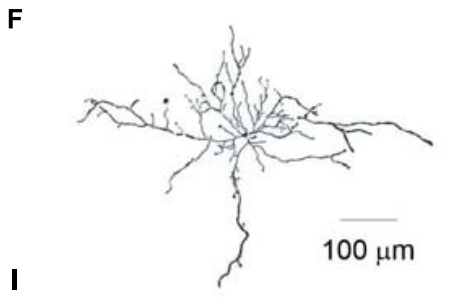

G

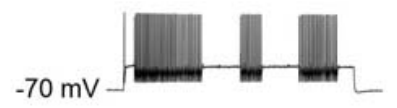

$\mathbf{J}$
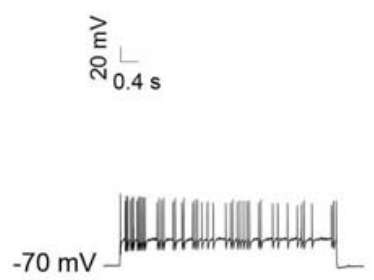

C

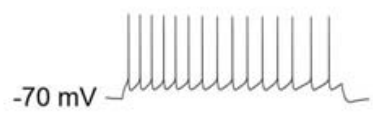

E

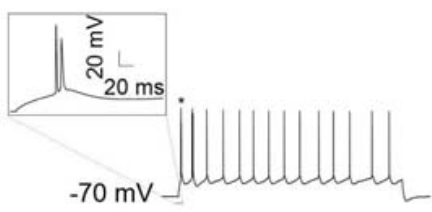

H

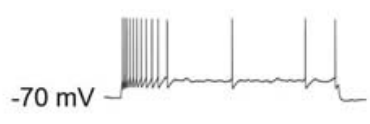

K



L

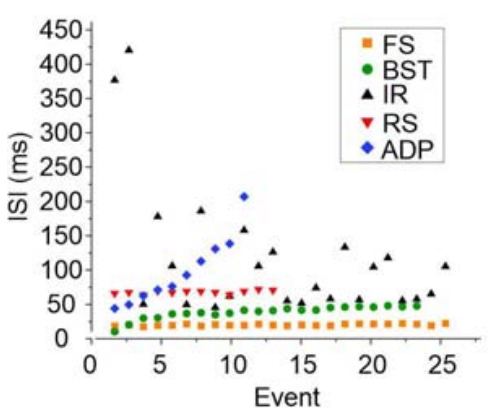

M

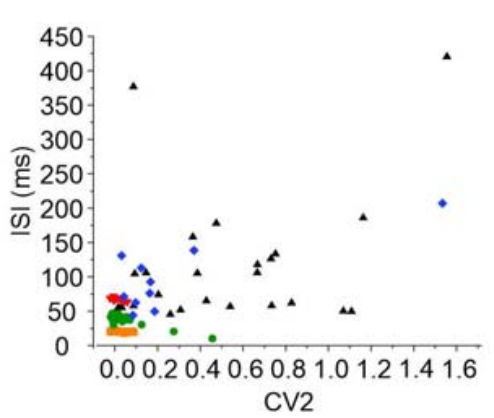

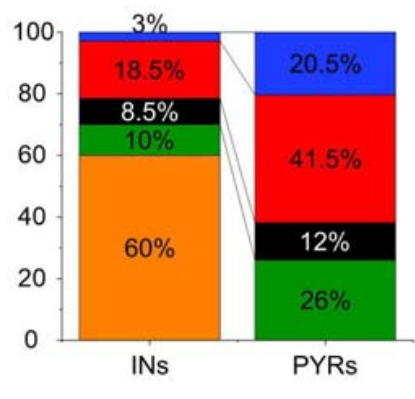

FIGURE 1 | Morphology and firing patterns of pyramidal neurons and GABAergic interneurons of the PRC. (A) Imaris reconstruction of a biocytin-labeled pyramidal neuron of the PRC. (B-E) Firing traces recorded from representative adapting (ADP) (B), late- and regular-spiking (RS) (C), irregular (IR) (D), and bursting (BST; burst magnification in the inset) (E) PRC pyramidal neurons. (F) Imaris reconstruction of a biocytin-labeled perirhinal GABAergic interneuron. (G-K) Firing traces recorded from representative stuttering fast-spiking (FS) (G), adapting (ADP) (H), late- and regular-spiking (RS) (I), irregular (IR) (J), and bursting (BST; burst magnification in the inset) (K) PRC GABAergic interneurons. (L,M) Inter-spike-interval (ISI) to event plot (L) and inter-spike-interval (ISI) to coefficient of variation (CV2) plot (M) showing the behavior of five representative cells, one for each type of firing patter identified in the PRC. (N) Percentage of each type of discharge in PRC pyramidal cells $(N=128)$ and GABAergic interneurons $(N=70)$, respectively.

area or layer (Table 3), suggesting that resonance could be very important for the oscillatory synchronization and integration of the neuronal activity in this region. Also, the resonance strength
$\left(Q_{-70}\right)$ and the frequency of resonance $\left(F_{\text {res }}\right)$ were similar in pyramidal neurons of A35 vs. A36 and of superficial vs. deep layers (Figures 3A-D). Comparable results were obtained also 
TABLE 2 | Comparison of the principal passive electrophysiological properties of pyramidal neurons and GABAergic interneurons of the PRC.

\begin{tabular}{lcccc}
\hline & $\begin{array}{c}\text { Number of } \\
\text { cells }(\mathbf{N})\end{array}$ & $\mathbf{C}_{\mathbf{m}}(\mathbf{p F})$ & $\mathbf{R}_{\text {in }}(\mathbf{M} \boldsymbol{\Omega})$ & $\mathbf{V}_{\mathbf{r}}(\mathbf{m V})$ \\
\hline Pyramidal neurons & 128 & $92.5 \pm 2.4$ & $181 \pm 5$ & $-67.8 \pm 0.5$ \\
Interneurons & 70 & $34.4 \pm 1.2 * * *$ & $210 \pm 10 * *$ & $-67.9 \pm 0.7$
\end{tabular}

Values of membrane capacitance, membrane input resistance, and membrane resting potential for each cell type are reported as mean \pm SEM and were statistically compared (pyramidal neurons vs. GABAergic interneurons) by unpaired Student's t-test. ${ }^{* *} p<0.01 ;{ }^{* * *} p<0.001$.

in GABAergic interneurons (Figures 3A-D). However, we found that the $Q_{-70}$ was significantly different between pyramidal neurons and GABAergic interneurons regardless of their location (Figures 3A,C).

In a subset of cells, experiments were repeated using "inverted" $(N=13)$ and "negative" $(N=12)$ ZAP protocols without any changes in the reported resonant properties of the cells (data not shown). This demonstrates that the resonant peak depends on the frequency of stimulation and not on the timing or orientation of the ZAP input. The ZAP input was also applied before and following perfusion of the synaptic blockers NBQX (10 $\mu \mathrm{M})$, (RS)-CPP (30 $\mu \mathrm{M})$, and bicuculline methiodide (10 $\mu \mathrm{M})$, without any changes in resonant properties demonstrating that membrane resonance is an intrinsic property of the neurons and is not driven by synaptic input $(N=5$; not shown).

\section{Correlations in Firing Properties, Passive Properties, and Resonant/Non-resonant Behavior of the PRC Neurons}

As already stated, PRC pyramidal neurons and GABAergic interneurons showed different firing patterns in response to the injection of suprathreshold current steps. Therefore, we tried to identify possible correlations between the firing pattern and the resonant/non-resonant behavior of these cells. To this purpose, we computed the percentage of specific firing patterns for nonresonant and resonant pyramidal (Figures 4A,B) and GABAergic neurons (Figures $\mathbf{4} \mathbf{A}^{\prime}, \mathbf{B}^{\prime}$ ). Furthermore, we computed the percentage of resonant vs. non-resonant cells classified for a specific firing pattern of pyramidal (Figure 4C) and GABAergic neurons (Figure $4 \mathbf{C}^{\prime}$ ). In pyramidal neurons, we found a prevalence of resonant neurons regardless of the firing pattern (Figure 4C). In addition, the percentage of the different types of firing pattern was quite similar in non-resonant (Figure 4A) and resonant (Figure $4 \mathbf{B}$ ) cells. In contrast, in GABAergic interneurons we found (i) a large prevalence of resonant cells in RS, BST, and ADP neurons, (ii) an equal percentage of resonant vs. non-resonant cells in IR neurons, and iii) a prevalence of non-resonant cells in FS neurons (Figure 4C'). Resonant interneurons showed all the types of firing patterns, although in different percentages (Figure $\mathbf{4} \mathbf{B}^{\prime}$ ). On the contrary, a high percentage $(84.5 \%)$ of non-resonant GABAergic interneurons were FS (Figure 4A' $\mathbf{A}^{\prime}$ ).

To clarify the interdependencies between passive properties, firing properties, and resonance in the neurons of the different PRC areas and layers, we plotted pairwise scatterplots of $\mathrm{C}_{\mathrm{m}}$ (membrane capacitance), $\mathrm{R}_{\mathrm{in}}$ (input resistance), $\mathrm{V}_{\mathrm{r}}$ (membrane resting potential), $\mathrm{AP}_{\mathrm{Th}}$ (spike Threshold), $\mathrm{AP}_{\mathrm{A}}$ (spike Amplitude), $\mathrm{AP}_{\mathrm{D}}$ (spike Duration), First ISI, Mean ISI, Mean CV2, Q-70 recorded from pyramidal and GABAergic neurons of A35 and A36 (Figure 5) and of superficial and deep layers (Figure 6) of the PRC. Then we computed Pearson's correlation coefficients for each of them. By examining separately pyramidal neurons or GABAergic interneurons, we found that the correlation matrices were quite similar among the different areas (Figure 5) and layers (Figure 6). As expected, we found significant correlations in pairwise comparisons of passive properties (for example inverse correlation between $\mathrm{C}_{\mathrm{m}}$ and $\mathrm{R}_{\text {in }}$ ) and of firing properties (for example inverse correlation between $\mathrm{AP}_{\mathrm{Th}}$ and $\mathrm{AP}_{\mathrm{A}}$; direct correlation between Mean ISI and Mean CV2).

Focusing on $\mathrm{Q}_{-70}$, in pyramidal neurons we found that this parameter was significantly correlated only with $R_{\text {in }}$ (inverse correlation) (Figures 5A-C, 6A,B). Furthermore, by directly comparing $\mathrm{R}_{\text {in }}$ in resonant vs. non-resonant pyramidal neurons, we found a significantly lower $R_{\text {in }}$ in resonant cells (Table 4). We also found a significantly more depolarized $V_{r}$ in resonant pyramidal neurons (Table 4). Overall, we assume these results to be due to the presence of a putative cationic current expressed only in resonant cells and active at their $\mathrm{V}_{\mathrm{r}}(-70 \mathrm{mV})$.

In GABAergic interneurons we found significant correlations between $\mathrm{Q}_{-70}$ and many other passive (direct correlations with $\mathrm{C}_{\mathrm{m}}, \mathrm{V}_{\mathrm{r}}$, and $\mathrm{R}_{\mathrm{in}}$ ) and firing (direct correlations with Mean ISI and $\mathrm{AP}_{\mathrm{D}}$ ) parameters (Figures $\mathbf{5} \mathbf{A}^{\prime}-\mathbf{C}^{\prime}, \mathbf{6} \mathbf{A}^{\prime}, \mathbf{B}^{\prime}$ ). This suggested an effective correlation between the firing patterns and the resonant/non-resonant behavior of the GABAergic interneurons, as already indicated by the percentage distributions in Figure 4. In fact, non-resonant GABAergic interneurons were mainly FS cells characterized, compared to the other subclasses of interneurons, by higher firing rate (lower Mean ISI), lower $A_{D}$, lower $C_{m}$, and lower $R_{\text {in }}$ (Ma et al., 2006). Interestingly, the direct comparison of $\mathrm{R}_{\text {in }}$ in resonant vs. non-resonant GABAergic interneurons reinforced this hypothesis since we found a significantly lower $\mathrm{R}_{\mathrm{in}}$ in non-resonant cells (Table 4). As in pyramidal neurons, we also found a significantly more depolarized $\mathrm{V}_{\mathrm{r}}$ in resonant GABAergic neurons (Table 4), likely due to both the higher $R_{\text {in }}$ and the expression of a resonant-specific cationic current.

\section{Resonance Properties at Different Membrane Potentials}

In resonant neurons, the voltage-dependence of $\mathrm{Q}$ and $\mathrm{F}_{\text {res }}$ was tested by applying the ZAP protocol at different membrane potentials, ranging from -55 to $-90 \mathrm{mV}$. In pyramidal neurons (solid line), resonance $(Q$-values $>1.05)$ began at potential more negative than $-55 \mathrm{mV}$ and was preserved at more hyperpolarized potentials (Figure 7A). Resonance strength was maximal at -70 $\mathrm{mV}$, as emphasized by the relative increase in the $\mathrm{Q}$-value at this potential $(\mathrm{Q}=1.24 \pm 0.02$, Figure 7A). 


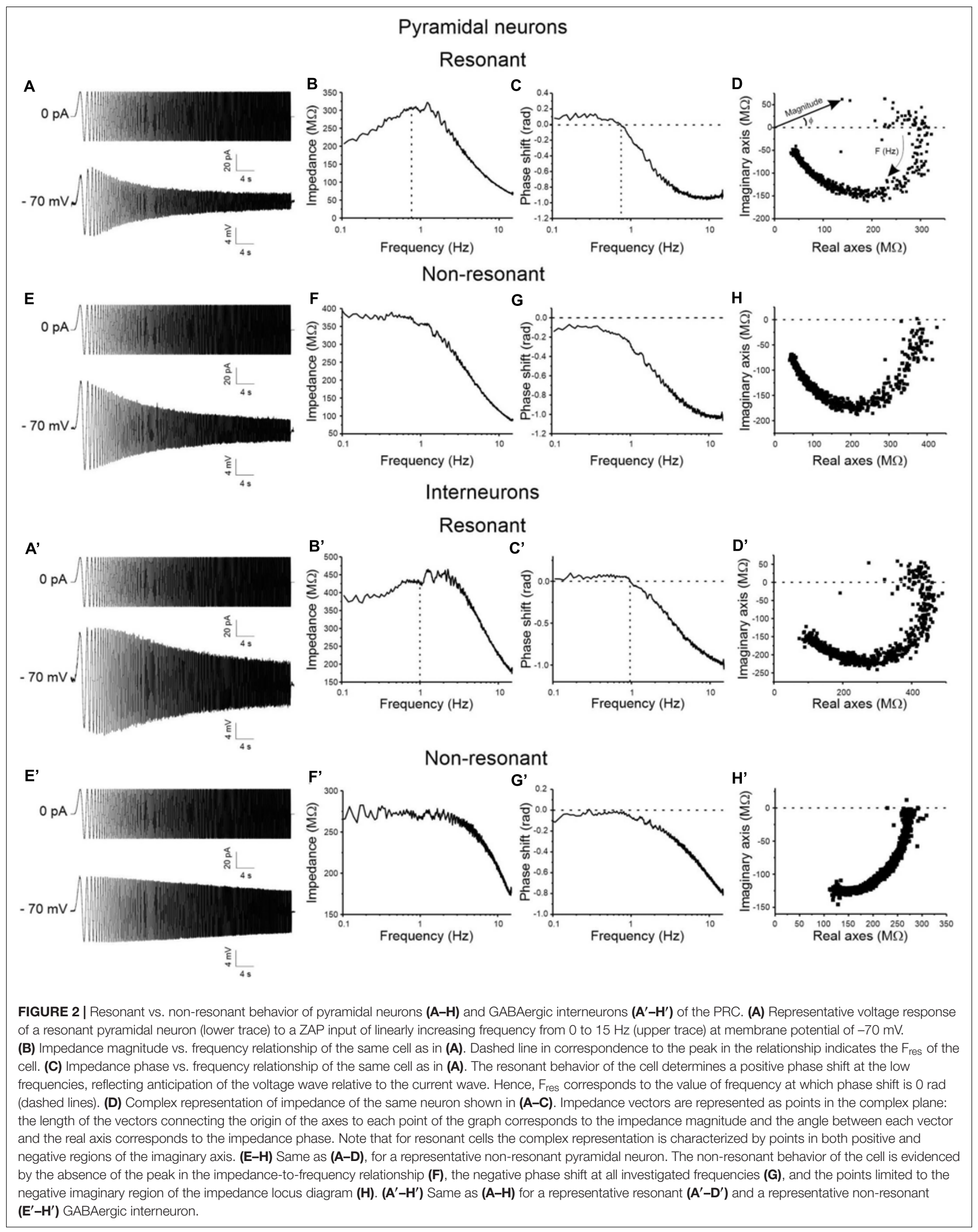


TABLE 3 | Distribution of the resonant pyramidal neurons and GABAergic interneurons in the superficial and deep layers of the areas 35 (A35) and 36 (A36) of the PRC.

\begin{tabular}{|c|c|c|c|c|c|c|c|c|}
\hline & \multicolumn{2}{|c|}{ A35 } & \multicolumn{2}{|l|}{ A36 } & \multicolumn{2}{|c|}{ Total } & \multicolumn{2}{|c|}{ Total } \\
\hline & Superficial layers & Deep layers & Superficial layers & Deep layers & A35 & A36 & Superficial layers & Deep layers \\
\hline Pyramidal neurons & $10 / 15$ (67\%) & 44/55 (80\%) & 9/17 (53\%) & $36 / 41$ (88\%) & $54 / 70(77 \%)$ & 45/58 (78\%) & 19/32 (59\%) & 80/96 (83\%) \\
\hline Interneurons & $6 / 12$ (50\%) & $20 / 40$ (50\%) & 4/5 (80\%) & $8 / 13(62 \%)$ & $26 / 52$ (50\%) & 12/19 (63\%) & 10/17 (59\%) & $28 / 53(53 \%)$ \\
\hline
\end{tabular}

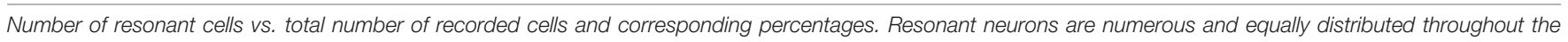
PRC, without a clear prevalence in a specific area or layer.

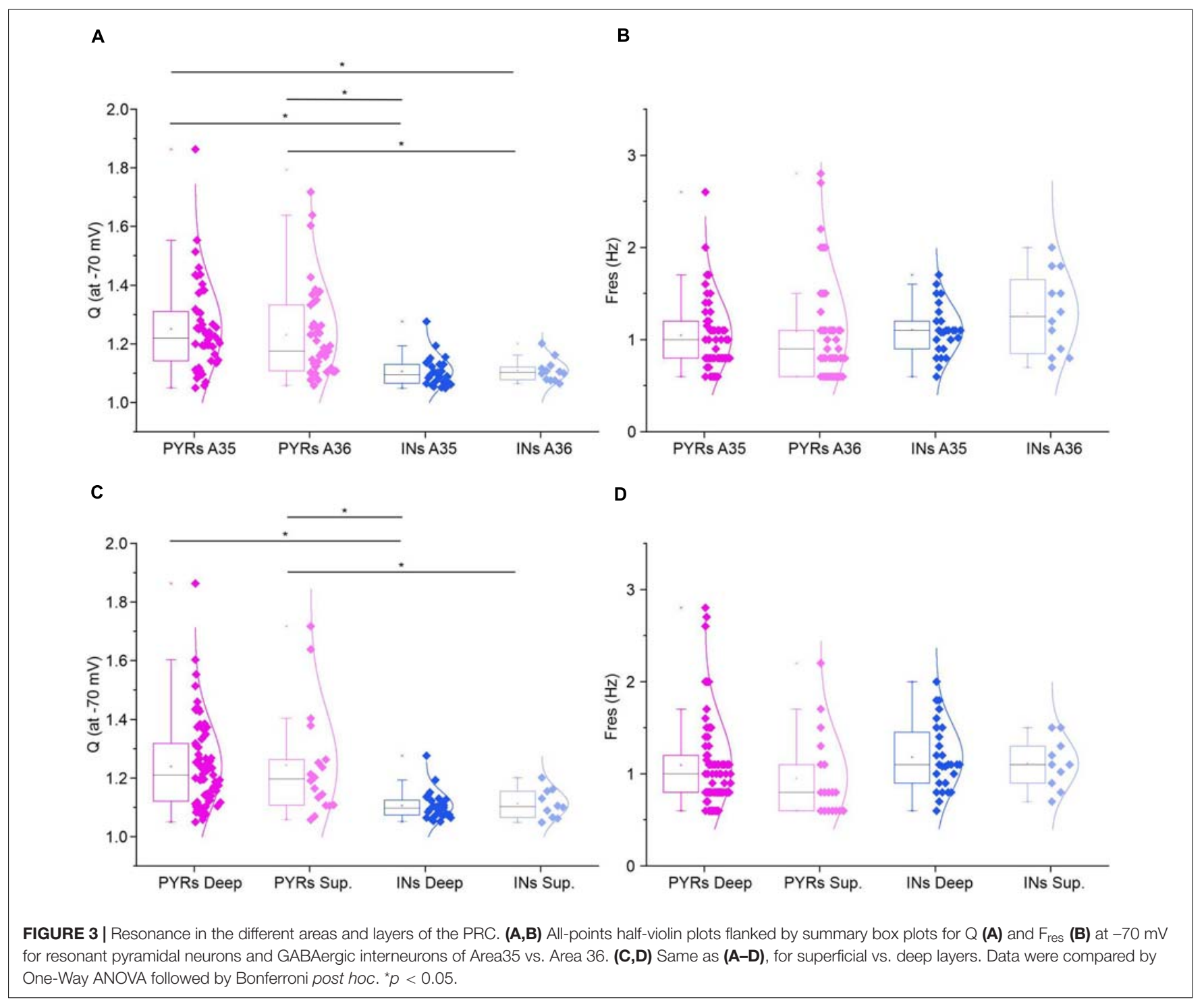

In contrast, GABAergic interneurons (dashed line) start to show resonance at potentials more negative than $-60 \mathrm{mV}$ and did not show a clear increase in the $Q$-value at any specific potential (Figure 7A). It is noteworthy that $Q$-values were significantly greater in pyramidal neurons than in GABAergic interneurons at both $-60 \mathrm{mV}(p<0.01)$ and $-70 \mathrm{mV}(p<0.001)$, perhaps suggesting that pyramidal neurons express an amplifying conductance that increases the $Q$-values which might be not present in interneurons.
$F_{\text {res }}$ was similar in the two cell types and nearly constant $(1-1.5 \mathrm{~Hz})$ in a wide range of membrane potentials (Figure $7 \mathbf{B}$ ).

\section{Effects of the Block of HCN Channels on Resonant Behavior of the Perirhinal Neurons}

As stated above, we found a more depolarized membrane resting potential in resonant compared to non-resonant neurons, 
A

PYRs non-resonant

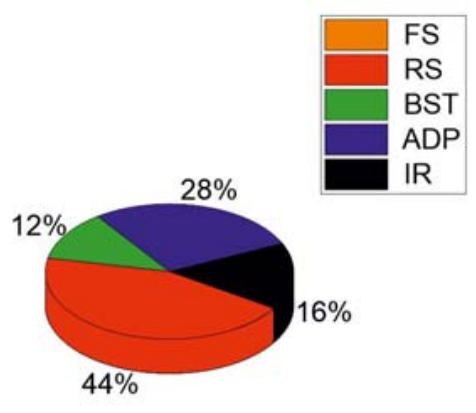

$A^{\prime}$

INs non-resonant

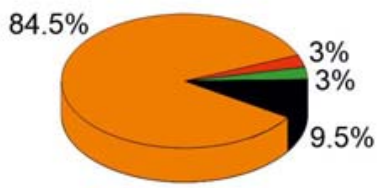

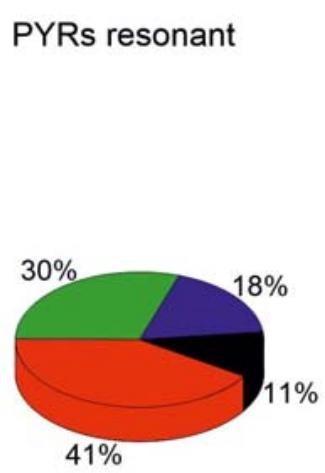

B'

INs resonant

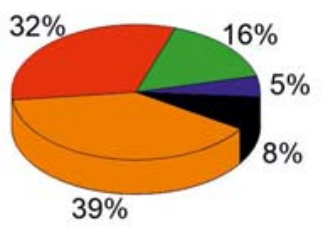

\section{C}

$\%$ Resonant

$\%$ Non-resonant

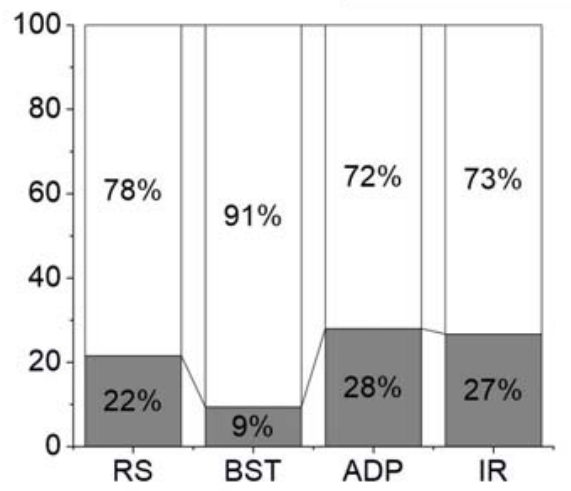

C'

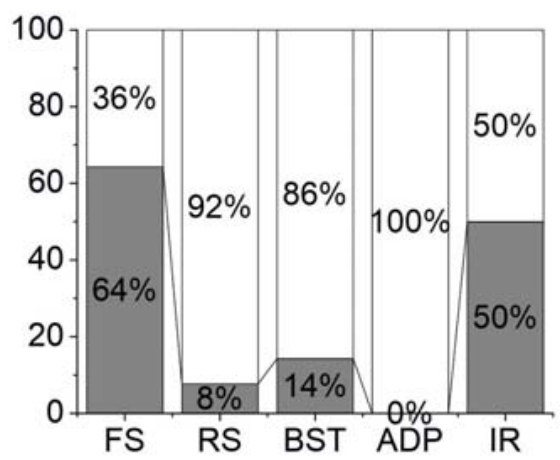

FIGURE 4 | Relationships between firing patterns and resonant or non-resonant behavior of the PRC neurons. (A,B) Percentage of specific firing patterns for resonant (A) and non-resonant (B) PRC pyramidal neurons. (C) Percentage of resonant vs. non-resonant cells classified for a specific firing pattern of pyramidal neurons. $\left(\mathbf{A}^{\prime}-\mathbf{C}^{\prime}\right)$ Same as $(\mathbf{A}-\mathbf{C})$ for GABAergic interneurons.

suggesting the presence of a putative cationic conductance expressed only in resonant cells and active at $\mathrm{V}_{\mathrm{r}}(-70 \mathrm{mV})$. Moreover, resonance behavior was preserved at hyperpolarized potentials, but disappeared at depolarized potentials. Based on this evidence and on literature (Hutcheon et al., 1996; Hu et al., 2002; Nolan et al., 2007; Biel et al., 2009; Ehrlich et al., 2012; Boehlen et al., 2013; Vera et al., 2014), we hypothesized the involvement of an $\mathrm{I}_{\mathrm{h}}$ current in the resonance of PRC neurons. $I_{h}$ is indeed a cationic subthreshold current showing biophysical properties that could cause low-frequency resonance at hyperpolarized potentials.

Consistently with the hypothesis that $\mathrm{I}_{\mathrm{h}}$ is implicated in PRC resonance, we found a prominent "sag" in the voltage response to hyperpolarizing current steps, likely due to $\mathrm{I}_{\mathrm{h}}$ activation, in all resonant perirhinal neurons (Figures $\mathbf{8 A}, \mathbf{A}^{\prime}$ ), but not in non-resonant neurons. The "sag" was completely abolished by the administration of ZD7288 $(10 \mu \mathrm{M})$, a specific blocker of HCN (hyperpolarization-activated cyclic nucleotide-gated) channels whose activation generate the $\mathrm{I}_{\mathrm{h}}$ current in neurons (Figures 8A, $\mathbf{A}^{\prime}$ ).

Following blockage of $\mathrm{I}_{\mathrm{h}}$ by ZD7288, the expression of resonant behavior was completely blocked (Figures $\mathbf{8 B}-\mathbf{E}, \mathbf{B}^{\prime}-\mathbf{E}^{\prime}$ ).
The effect of the inhibition of $I_{h}$ on the ZAP response is shown in the example traces reported in Figures 8B, $\mathbf{B}^{\prime}$. Following ZD7288 application the peak in the impedance-to-frequency relationship was abolished (Figures 8C, $\mathrm{C}^{\prime}$ ) and the $Q$-value decreased below the resonance threshold (Figures $\mathbf{8 D}, \mathbf{D}^{\prime}$ ) for both resonant pyramidal cells and GABAergic interneurons, confirming a key role of $\mathrm{I}_{\mathrm{h}}$ in generating resonance in PRC.

\section{Role of Persistent Sodium Current in Amplifying the Resonant Behavior of Perirhinal Pyramidal Neurons}

Given that the $Q$-value was significantly higher in resonant pyramidal neurons when compared to resonant interneurons at -60 and $-70 \mathrm{mV}$ (Figure 7A), we hypothesized that these neurons selectively express an amplifying current that is widely described in several classes of resonant cells (Traub and Miles, 1991; Gutfreund et al., 1995; Hutcheon et al., 1996; Hutcheon and Yarom, 2000; Hu et al., 2002; Vera et al., 2014; Matsumoto-Makidono et al., 2016). Since the persistent sodium current $\left(\mathrm{I}_{\mathrm{NaP}}\right)$ is a typical amplifying current that has been already reported to enhance the resonant behavior at 


\section{Pyramidal neurons}

A A35

B

A36
C

A35+A36

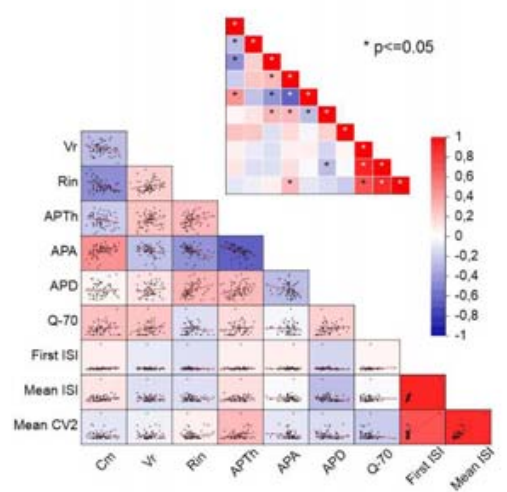

$A^{\prime}$

A35

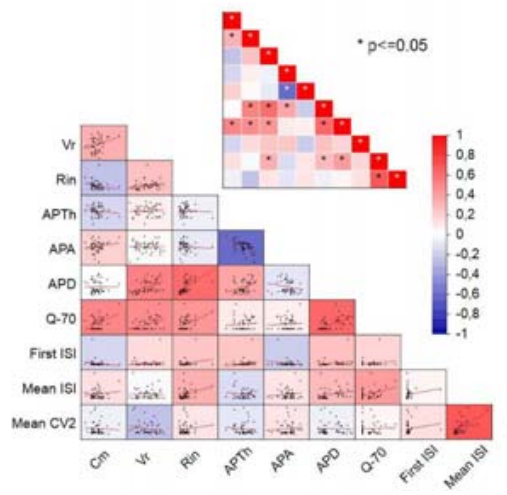

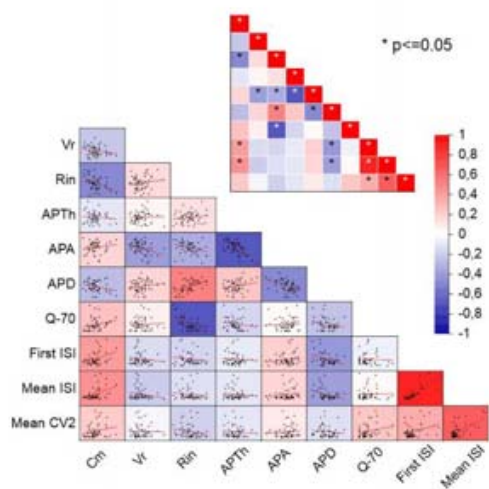

Interneurons

B'

A36

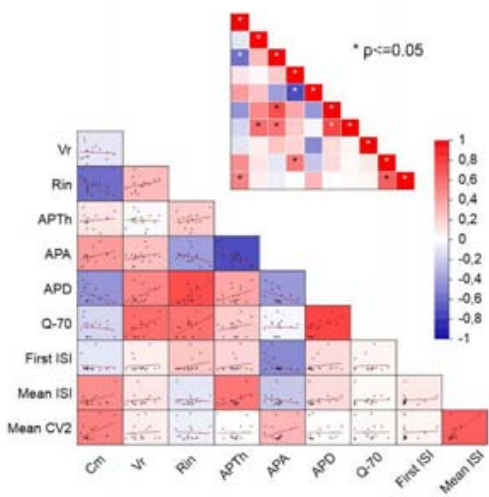

C'

A35+A36

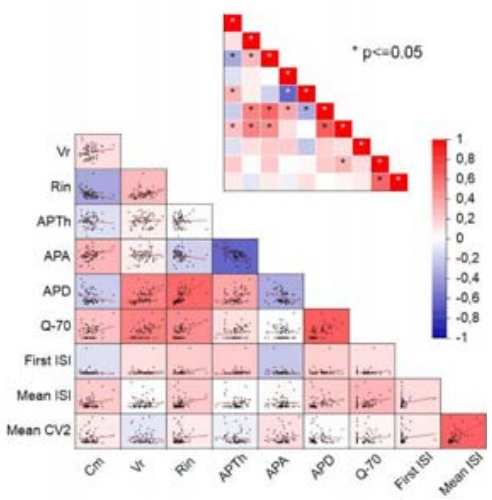

FIGURE 5 | Correlations between passive properties, firing properties, and resonance in the neurons of the different PRC areas. (A-C) Pairwise scatterplot matrices of $\mathrm{C}_{m}$ (membrane capacitance), $\mathrm{V}_{\mathrm{r}}$ (membrane resting potential), $\mathrm{R}_{\text {in }}$ (input resistance), $A P_{T h}$ (spike Threshold), $A P_{A}$ (spike $\left.A m p l i t u d e\right), A P_{D}$ (spike Duration), $Q_{-70}$, First ISI, Mean ISI, Mean CV2 of pyramidal neurons recorded from A35 (A), A36 (B), and both A35+A36 (C). These scatterplot matrices are overlaid on the corresponding color-coded correlation matrices and the insets in each panel represent the significance value associated with each scatterplot, computed as Pearson's correlation coefficients. ( $\left.\mathbf{A}^{\prime}-\mathbf{C}^{\prime}\right)$ Same as $(\mathbf{A}-\mathbf{C})$ for GABAergic interneurons. ${ }^{*} p \leq 0.05$.

membrane potentials near $\mathrm{V}_{\mathrm{r}}$ (Hutcheon et al., 1996; Vera et al., 2014), we tested the involvement of $\mathrm{I}_{\mathrm{NaP}}$ on the amplification of the membrane resonance in PRC pyramidal neurons. To this purpose, we applied TTx $(1 \mu \mathrm{M})$ during the administration of the ZAP protocol. We found that TTx attenuates the resonance strength measured at $-70 \mathrm{mV}$ in pyramidal neurons (Figures 9A,B) but not in GABAergic interneurons (Figures $\mathbf{9} \mathbf{A}^{\prime}, \mathbf{B}^{\prime}$ ). Accordingly, the $Q$-value was significantly reduced from 1.3 to 1.2 in pyramidal cells $(p<0.01)$ (Figure 9C), whereas it remained unchanged in GABAergic interneurons (Figure 9C'). As expected, TTx did not alter the values of $\mathrm{F}_{\text {res }}$ in both cell type (Figures $9 \mathrm{D}, \mathrm{D}^{\prime}$ ).

These data confirm that the persistent sodium current has an amplifying effect on the resonant behavior specifically in the pyramidal neurons of the PRC. TTx blocks also the transient
$\mathrm{Na}^{+}$current but we excluded the involvement of this current in resonance amplification because of its fast kinetics and its more depolarized activation threshold.

\section{Effect of Temperature on the Resonance Properties of Perirhinal Neurons}

It has been shown that the resonance properties of the neurons can be affected by temperature ( $\mathrm{Hu}$ et al., 2002; Yan et al., 2012; Vera et al., 2014). To verify whether the resonance properties of perirhinal neurons are also influenced by temperature, we performed a set of experiments at $36^{\circ} \mathrm{C}$. Figures $10 \mathrm{~A}, \mathbf{A}^{\prime}$ show representative impedance profiles derived at $36^{\circ} \mathrm{C}$ from a resonant pyramidal neuron and a resonant GABAergic interneuron, respectively. Data were obtained by 


\section{Pyramidal neurons}

A

\section{Deep layers}

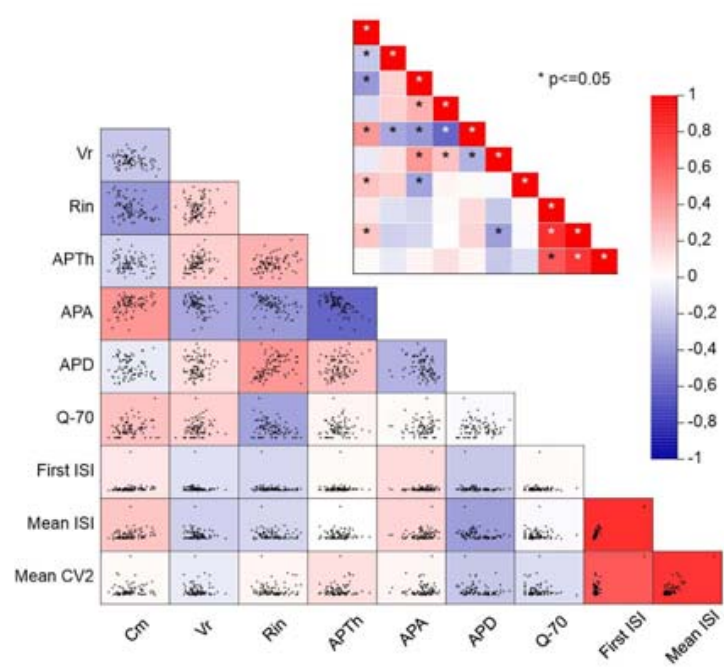

B

Superficial layers

\section{Interneurons}

A' B'

Deep layers

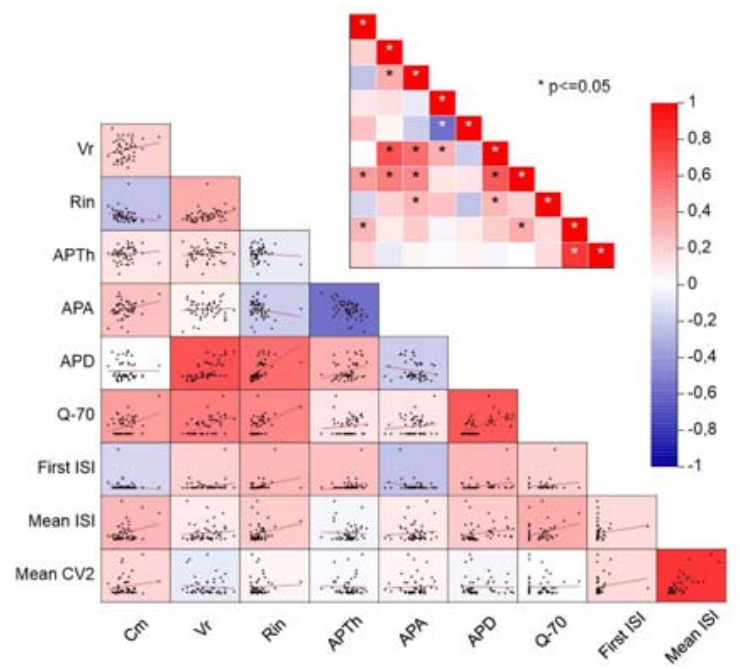

\section{Superficial layers}

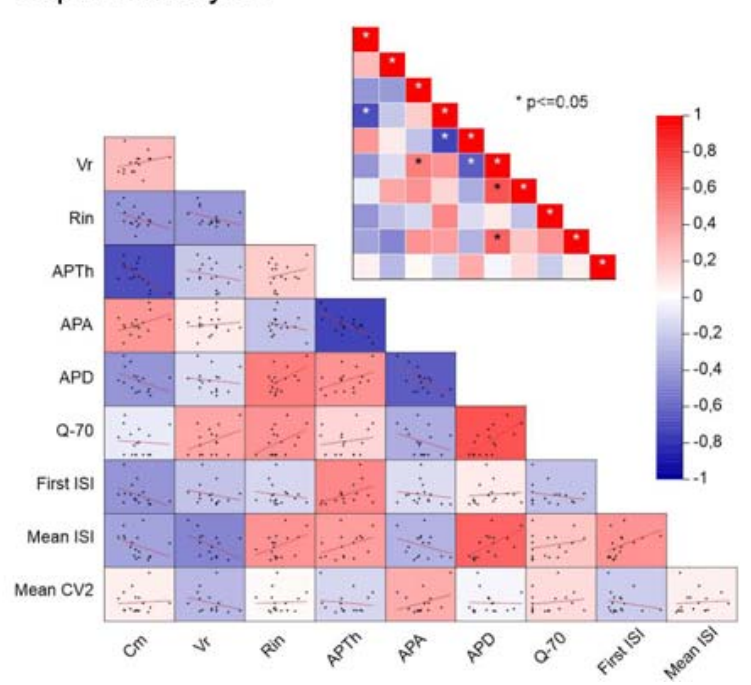

FIGURE 6 | Correlations between passive properties, firing properties, and resonance in the neurons of the different PRC layers. (A,B) Pairwise scatterplot matrices of $C_{m}$ (membrane capacitance), $V_{r}$ (membrane resting potential), $R_{\text {in }}$ (input resistance), $A P_{\text {Th }}$ (spike Threshold), $A P_{A}$ (spike $\left.A m p l i t u d e\right), A P_{D}$ (spike Duration), $Q-70$, First ISI, Mean ISI, Mean CV2 of pyramidal neurons recorded from the deep (A) and the superficial (B) layers. These scatterplot matrices are overlaid on the corresponding color-coded correlation matrices and the insets in each panel represent the significance value associated with each scatterplot, computed as Pearson's correlation coefficients. $\left(\mathbf{A}^{\prime}, \mathbf{B}^{\prime}\right)$ Same as $\mathbf{( A , B )}$ for GABAergic interneurons. ${ }^{*} p \leq 0.05$.

applying the standard $0-15 \mathrm{~Hz}$ ZAP protocol at $-70 \mathrm{mV}$. The analysis revealed that the $Q$-value does not change by increasing the temperature from 23 to $36^{\circ} \mathrm{C}$, either in pyramidal neurons or GABAergic interneurons (Figures $\mathbf{1 0 B}, \mathbf{B}^{\prime}$ ). On the contrary, resonant frequency significantly increased from $1.17 \pm 0.04$ to $2.53 \pm 0.49 \mathrm{~Hz}$ in pyramidal neurons $(p<0.05)$ and from
$1.13 \pm 0.04$ to $2.00 \pm 0.22 \mathrm{~Hz}$ in GABAergic interneurons $(p<0.05)$ (Figures 10C, $\left.\mathbf{C}^{\prime}\right)$.

\section{Computational Model}

The resonant behavior of the perirhinal pyramidal neurons and GABAergic interneurons was reproduced by a computational 
TABLE 4 | Comparison of the principal passive electrophysiological properties of resonant vs. non-resonant neurons of the PRC.

\begin{tabular}{|c|c|c|c|c|c|c|c|c|}
\hline & \multicolumn{2}{|c|}{ Number of cells $(\mathbf{N})$} & \multicolumn{2}{|c|}{$C_{m}(p F)$} & \multicolumn{2}{|c|}{$\mathbf{R}_{\mathrm{in}}(\mathrm{M} \Omega)$} & \multicolumn{2}{|c|}{$V_{r}(m V)$} \\
\hline & Resonant & Non-resonant & Resonant & Non-resonant & Resonant & Non-resonant & Resonant & Non-resonant \\
\hline Pyramidal neurons & 99 & 29 & $94.5 \pm 2.5$ & $85.8 \pm 6.3$ & $175 \pm 5^{*}$ & $202 \pm 15$ & $-67.1 \pm 0.5^{* *}$ & $-70.3 \pm 1.0$ \\
\hline Interneurons & 38 & 32 & $35.7 \pm 1.9$ & $32.9 \pm 1.3$ & $237 \pm 15^{* *}$ & $177 \pm 9$ & $-65.9 \pm 0.8^{\star \star \star}$ & $-70.3 \pm 0.9$ \\
\hline
\end{tabular}

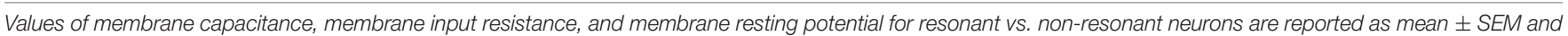
were statistically compared by unpaired Student's t-test. ${ }^{*} p<0.05 ;{ }^{* *} p<0.01$; ${ }^{* * *} p<0.001$.
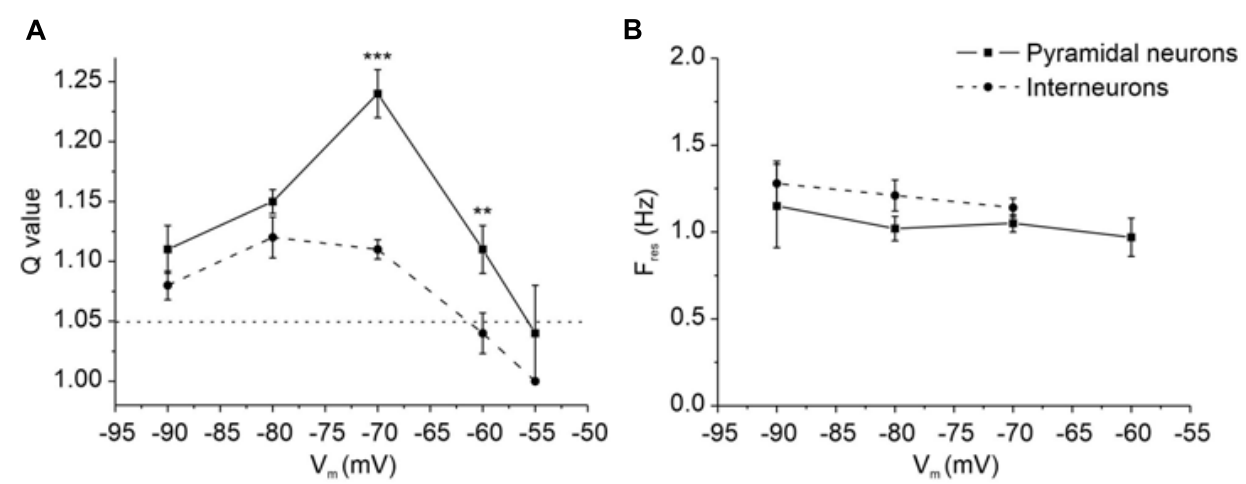

FIGURE 7 | Resonance properties of PRC pyramidal neurons and GABAergic interneurons at different membrane potentials. (A) Average Q values at different membrane potentials for pyramidal neurons (solid line) and GABAergic interneurons (dashed line). $Q$-value is defined as the ratio of impedance magnitude at $F_{\text {res }}$ and $0.1 \mathrm{~Hz}$ and is used to highlight the strength of the resonance. Dotted line at $Q=1.05$ discriminates between absence $(Q-v a l u e<1.05)$ and presence ( $Q$-value $\geq 1.05$ ) of resonance. On average, pyramidal neurons show a significantly higher $Q$-value at -60 and $-70 \mathrm{mV}(N=35$ and $N=99$, respectively) compared to GABAergic interneurons recorded at the same membrane potentials ( $N=15$ and $N=37$, respectively) $\left({ }^{\star \star} p<0.01\right.$; ${ }^{\star \star \star} p<0.001$; unpaired Student's $t$-test). Moreover, among pyramidal neurons the mean $Q$-value was greater at $-70 \mathrm{mV}(N=99)$ compared to the most of other tested potential $[-55 \mathrm{mV}(\rho<0.01, N=5)$, $-60 \mathrm{mV}(p<0.001, N=35),-80 \mathrm{mV}(p<0.001, N=40)$; Kruskal-Wallis followed by Dunn's multiple comparisons test]. Among interneurons the mean $Q$-value was lower at $-60 \mathrm{mV}(\mathrm{N}=15)$ compared to $-70 \mathrm{mV}(p<0.001, N=37)$ and $-80 \mathrm{mV}(p<0.01, N=12)$ (Kruskal-Wallis followed by Dunn's multiple comparisons test). (B) Average $F_{\text {res }}$ at different membrane potentials for pyramidal neurons (solid line) and GABAergic interneurons (dashed line). The mean values of $F_{\text {res }}$ are similar between pyramidal neurons [-60 mV ( $N=21),-70 \mathrm{mV}(\mathrm{N}=99),-80 \mathrm{mV}(\mathrm{N}=38)$ and $-90 \mathrm{mV}(\mathrm{N}=8)]$ and GABAergic interneurons $[-70 \mathrm{mV}(\mathrm{N}=37),-80 \mathrm{mV}$ $(N=12),-90 \mathrm{mV}(N=9)]$ at all tested potentials (unpaired Student's $t$-test).

model in MATLAB (see section "Materials and Methods" for details) by applying the same sinusoidal ZAP input current used in the electrophysiological experiments (Origin 6.0).

At first, the model was tested with a ZAP protocol at $-70 \mathrm{mV}$ in its basal conditions where only the leakage current $\left(\mathrm{I}_{\text {leak }}\right)$ was expressed (Figure 11A). The corresponding impedance profile (Figure 11B) strongly resembled the pattern of a nonresonant neuron acting as a low-pass filter. By adding the persistent sodium current $\left(\mathrm{I}_{\mathrm{NaP}}\right)$, the voltage response and the corresponding impedance profile still showed a lack of a resonant behavior (Figures 11C,D) indicating that $\mathrm{I}_{\mathrm{NaP}}$ alone is not sufficient to generate resonance. A resonant response was obtained by simulating the expression of the h-current $\left(\mathrm{I}_{h}\right)$, as indicated by the appearance of a peak in the impedance profile $(Q=1.1)$ (Figures $1 \mathbf{1 E}, \mathbf{F})$. The $F_{\text {res }}$ calculated from the impedance profile obtained in the model overlapped that measured during patch-clamp recording experiments. In a model simulating the presence of $\mathrm{I}_{\text {leak }}, \mathrm{I}_{\mathrm{h}}$, and $\mathrm{I}_{\mathrm{NaP}}$ an amplification of the resonance was observed (Figures 11G,H), as demonstrated by an increase of the $Q$-value $(Q=1.2)$ compared to the previous condition.

To examine whether the effect of the temperature on the resonant frequency was also reproducible in the model, the value of the simulated temperature (T) was increased up to $36^{\circ} \mathrm{C}$ (Figures $1 \mathbf{1 A}^{\prime}-\mathbf{H}^{\prime}$ ). As expected, $\mathrm{F}_{\text {res }}$ shifted toward higher values in the model simulating the presence of both

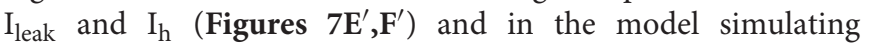
the contribution of all three currents $I_{\text {leak }}+I_{h}+I_{N a P}$

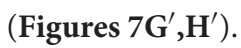

In summary, by implementing a computational model with parameters obtained with patch-clamp experiments we were able to reproduce the resonant behavior observed in both pyramidal neurons and GABAergic interneurons of the PRC.

\section{DISCUSSION AND CONCLUSION}

This paper describes for the first time the membrane resonance properties of pyramidal cells and GABAergic interneurons throughout the different layers of the mouse perirhinal cortex. These findings represent a missing piece of a comprehensive puzzle designed to define the functional contribution of the parahippocampal cortical regions in the processing of information directed to and propagated from the hippocampus. In particular, by applying a $0-15 \mathrm{~Hz}$ ZAP protocol, we demonstrated that a substantial subpopulation (over $75 \%$ of 


\section{Pyramidal neurons}


Interneurons
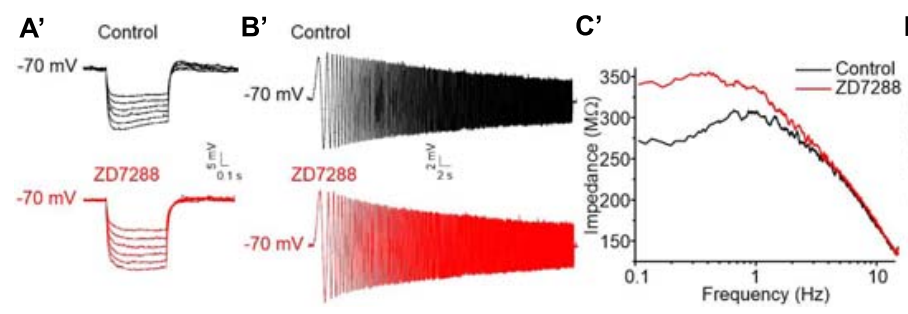

D'

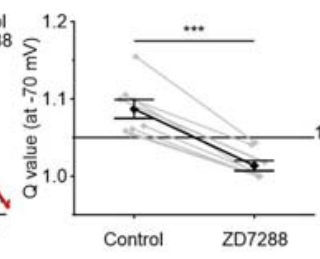

$E^{\prime}$

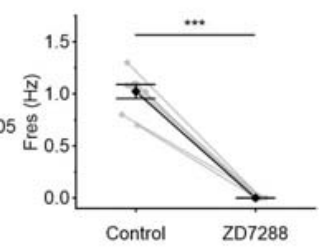

FIGURE 8 | Effects of the block of HCN channels on resonant behavior of pyramidal neurons (A-D) and GABAergic interneurons ( $\left(\mathbf{A}^{\prime}-\mathbf{D}^{\prime}\right)$ of the PRC. (A) Representative voltage traces evoked by applying hyperpolarizing current steps to a resonant pyramidal neuron. A prominent "sag" appears in the voltage responses (upper traces in black) and is abolished after administration of the HCN blocker ZD7288 $10 \mu \mathrm{M}$ (lower traces in red). (B) Voltage responses of a representative resonant pyramidal neuron to the standard 0-15 Hz ZAP protocol at $-70 \mathrm{mV}$ before (upper trace in black) and after (lower trace in red) the perfusion of ZD7288 $10 \mu \mathrm{M}$. (C) Impedance-to-frequency relationship of the same cell as in (B), before (black line) and after (red line) ZD7288 perfusion. (D,E) Scatter plots comparing the $Q$-values (C) and $F_{\text {res }}$ values (D) derived from resonant pyramidal neurons $(N=21)$ before (Control) and after (ZD7288) blocking the HCN channels (gray dots and lines). Mean \pm SEM. Q-values are reported on the same plot in black. In ZD7288 condition, the resonant behavior is completely abolished. ((A'-D') Same as (A-D) for GABAergic interneurons $(N=8)$ : also in GABAergic interneurons ZD7288 administration completely abolishes "sag" ( $\mathbf{A}^{\prime}$ ) and resonant behavior $\left(\mathbf{B}^{\prime}-\mathbf{D}^{\prime}\right)$. Data in $\left(\mathbf{D}, \mathbf{E}, \mathbf{D}^{\prime}, \mathbf{E}^{\prime}\right)$ were compared by paired Student's $t$-test. ${ }^{* \star *} p<0.001$.

pyramidal cells and over $50 \%$ of GABAergic interneurons) of the PRC neurons showed membrane resonance in a frequency $\left(\mathrm{F}_{\text {res }}\right)$ ranging from 1 to $2.5 \mathrm{~Hz}$. By using "inverted" and "negative" ZAP protocols, as well as by repeating the experiments before and following perfusion of synaptic blockers NBQX, (RS)-CPP, and bicuculline methiodide, we demonstrated that resonant behavior and peak are intrinsic properties of the PRC cells, not influenced by the time and the orientation of the ZAP input and not driven by synaptic input. It is worth noticing that bicuculline methiodide blocks also SK-channels (Johnson and Seutin, 1997). This is a potential caveat that needs to be considered, since SK-current could possibly be implicated in the generation of resonance (Xue et al., 2012). However, we did not observe changes in resonant properties following the perfusion of the synaptic blockers (including bicuculline methiodide). Then, we believe that SK-current is not implicated in the generation of the resonant response in the PRC cells and the results of the subset of experiments involving the administration of synaptic blockers are reliable.

We did not observed differences in the distribution of the pyramidal and GABAergic resonant neurons throughout the different areas (A35 and A36) and layers (deep and superficial) of the PRC. Furthermore, the resonance strength and the frequency of resonance seemed to be independent from the localization of the cells. The Pearson's correlation matrices of $\mathrm{C}_{\mathrm{m}}$ (membrane capacitance), $\mathrm{R}_{\mathrm{in}}$ (input resistance), $\mathrm{V}_{\mathrm{r}}$ (membrane resting potential), $\mathrm{AP}_{\mathrm{Th}}$ (spike Threshold), $\mathrm{AP}_{\mathrm{A}}$ (spike Amplitude), $\mathrm{AP}_{\mathrm{D}}$ (spike Duration), First ISI, Mean ISI, Mean CV2, and Q-70 were also similar when computed in different PRC areas and layers.

On the other hand, by analyzing possible correlations between the firing pattern and the resonant/non-resonant behavior of the PRC cells, we found interesting differences between pyramidal neurons and GABAergic interneurons. In pyramidal neurons the percentage of the different types of firing pattern (RS, ADP, BST, IR) was quite similar in non-resonant and resonant cells, with a majority of resonant cells regardless of the firing pattern. In pyramidal neurons, the $Q_{-70}$ was inversely correlated with $\mathrm{R}_{\text {in }}$. $\mathrm{R}_{\text {in }}$ was also significantly lower in cells that showed a resonant behavior and expressed the $I_{h}$ at $V_{r}$. No others significant correlations emerged. In contrast, in GABAergic interneurons Q-70 significantly correlated with many other passive (direct correlations with $\mathrm{C}_{\mathrm{m}}, \mathrm{V}_{\mathrm{r}}$, and $\mathrm{R}_{\text {in }}$ ) and firing (direct correlations with Mean ISI and $\mathrm{AP}_{\mathrm{D}}$ ) parameters. The high prevalence of FS cells-characterized by higher firing rate (lower Mean ISI), lower $\mathrm{AP}_{\mathrm{D}}$, lower $\mathrm{C}_{\mathrm{m}}$, and lower $\mathrm{R}_{\text {in }}$ (Ma et al., 2006) - in non-resonant GABAergic interneurons could support these correlations. A significantly lower $R_{i n}$ in non-resonant compared to resonant interneurons reinforced this hypothesis. Overall, these data suggested an effective correlation between the firing pattern and the resonant/non-resonant behavior in the GABAergic interneurons, but not in the pyramidal neurons of the PRC. 


\section{Pyramidal neurons}

A


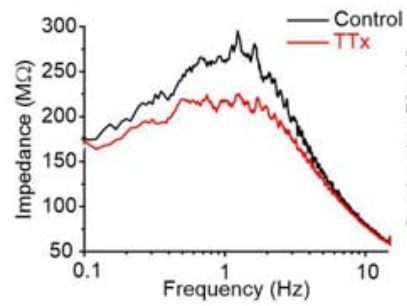

C

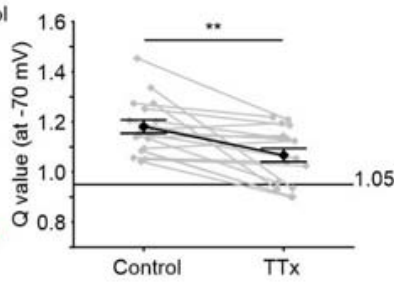

Interneurons

B'

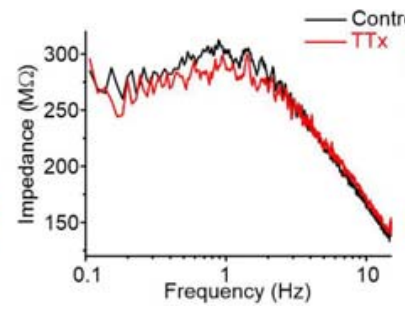

$c^{\prime}$

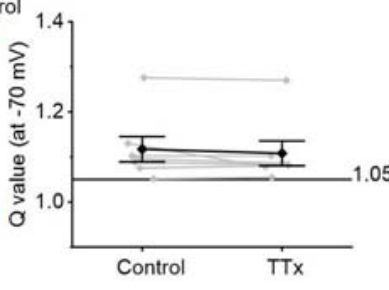

D

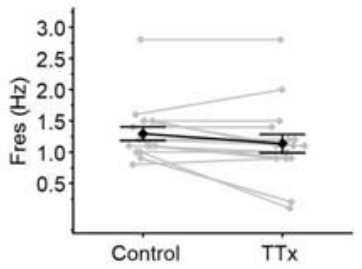

D'

FIGURE 9 | Effects of the block of $\Pi$ X-sensitive sodium channels on resonant behavior of pyramidal neurons (A-D) and GABAergic interneurons ( $\mathbf{A}^{\prime}$ - $\mathbf{D}^{\prime}$ ) of the PRC. (A) Voltage responses of a representative resonant pyramidal neuron to the standard $0-15 \mathrm{~Hz} Z \mathrm{ZAP}$ protocol at $-70 \mathrm{mV}$ before (upper trace in black) and after (lower trace in red) the perfusion of $T \mathrm{x} 1 \mu \mathrm{M}$. (B) Impedance-to-frequency relationship of the same cell as in (B), before (black line) and after (red line) $\Pi \mathrm{X}$ perfusion. (C,D) Scatter plots comparing the $Q$-values (C) and $F_{\text {res }}$ values (D) derived from resonant pyramidal neurons $(N=17)$ before $(C o n t r o l)$ and after (TTx) blocking the $\Pi$ Tx-sensitive Sodium channels (gray dots and lines). Mean \pm SEM. Q-values are reported on the same plot in black. In TTx condition, the Q-value is significantly

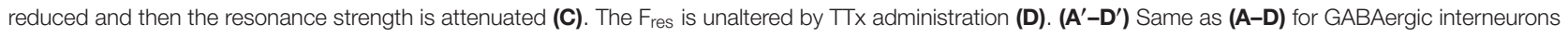
$(N=7)$. In GABAergic interneurons $T$ Tx perfusion has no effect either on the $Q$-value $\left(\mathbf{C}^{\prime}\right)$ or on the $F_{\text {res }}\left(\mathbf{D}^{\prime}\right)$. Data in $\left(\mathbf{C}, \mathbf{D}, \mathbf{C}^{\prime}, \mathbf{D} \mathbf{D}^{\prime}\right)$ were compared by paired Student's t-test. ${ }^{* *} p<0.01$.

Concerning voltage-dependence of resonance, the analysis of $\mathrm{F}_{\text {res }}$ at different membrane potential ranging from -90 to -55 $\mathrm{mV}$ showed very similar values. However, the characterization of the resonance strength at different holding membrane potentials, between -90 and $-55 \mathrm{mV}$, indicated that the resonant neurons manifest a voltage-dependent resonance. In pyramidal neurons the $Q$-value exhibited a peak at around $-70 \mathrm{mV}$, whereas in interneurons the maximum $Q$-value was measured between -80 and $-70 \mathrm{mV}$. These observations indicated a prominent response at potential value at or just below resting membrane potential. The abolishment of resonance in PRC neurons following $\mathrm{I}_{\mathrm{h}}$ current block with ZD7288 suggests that the expression of the $\mathrm{HCN}$ channels is mandatory for the definition of the resonant mechanism in PRC in both groups of neurons tested, confirming the important role played by HCN in the subthreshold resonance, as already demonstrated for other neocortical (Hutcheon et al., 1996) and limbic areas (Shay et al., 2012). The expression of this conductance only in resonant cells could also justify the more depolarized membrane resting potential of resonant compared to non-resonant neurons. Interestingly, the application of TTx caused a decrease in the resonant peak, but did not abolish the resonant behavior, suggesting that in pyramidal neurons of the
PRC a persistent sodium current is able to amplify the resonance produced by other conductances, as demonstrated in other brain areas. Indeed, at membrane potentials ranging between -70 and $-60 \mathrm{mV}$ the persistent sodium current produced in pyramidal neurons a significant amplification of the resonant peak that became steeper and sharpened, whereas the $F_{\text {res }}$ was not affected by the application of TTx. By contrast, in interneurons the amplification of the resonance mediated by persistent sodium current was not observed, probably due to a lack or to a low density of $\mathrm{I}_{\mathrm{NaP}}$ channels in these cells, as shown in CA1 (Hedrich et al., 2014) and in neocortex (Aracri et al., 2006). Alternatively, it could be hypothesized, in the case of interneurons, a concurrent activation of persistent sodium current together with a current, such as the potassium inward rectifier, that attenuates resonance. The experimental data were replicated by using a MatLab model, containing $\mathrm{I}_{\text {leak }}, \mathrm{I}_{\mathrm{h}}$ and $\mathrm{I}_{\mathrm{NaP}}$ channels. Currents' kinetics and gating properties were derived by literature (Hodgkin and Huxley, 1952; Spain et al., 1987), due to the lack of data describing these properties in the PRC pyramidal and GABAergic neurons. To overcome the limitations of the model and for a future upgrade, these parameters could be derived directly from PRC neurons. However, since these 


\section{Pyramidal neurons}

A

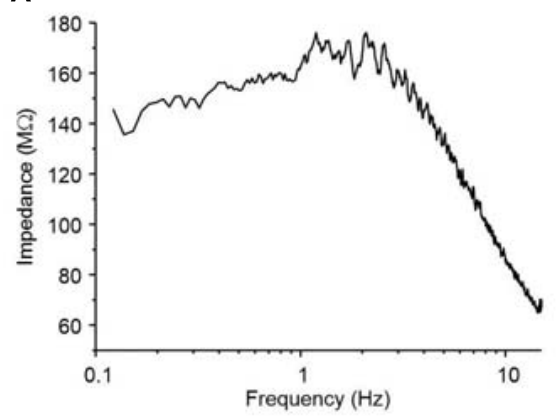

B

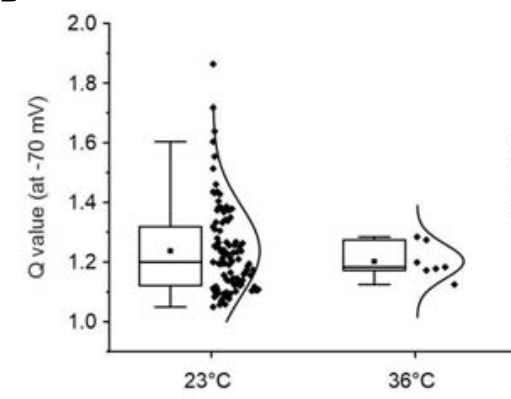

Interneurons
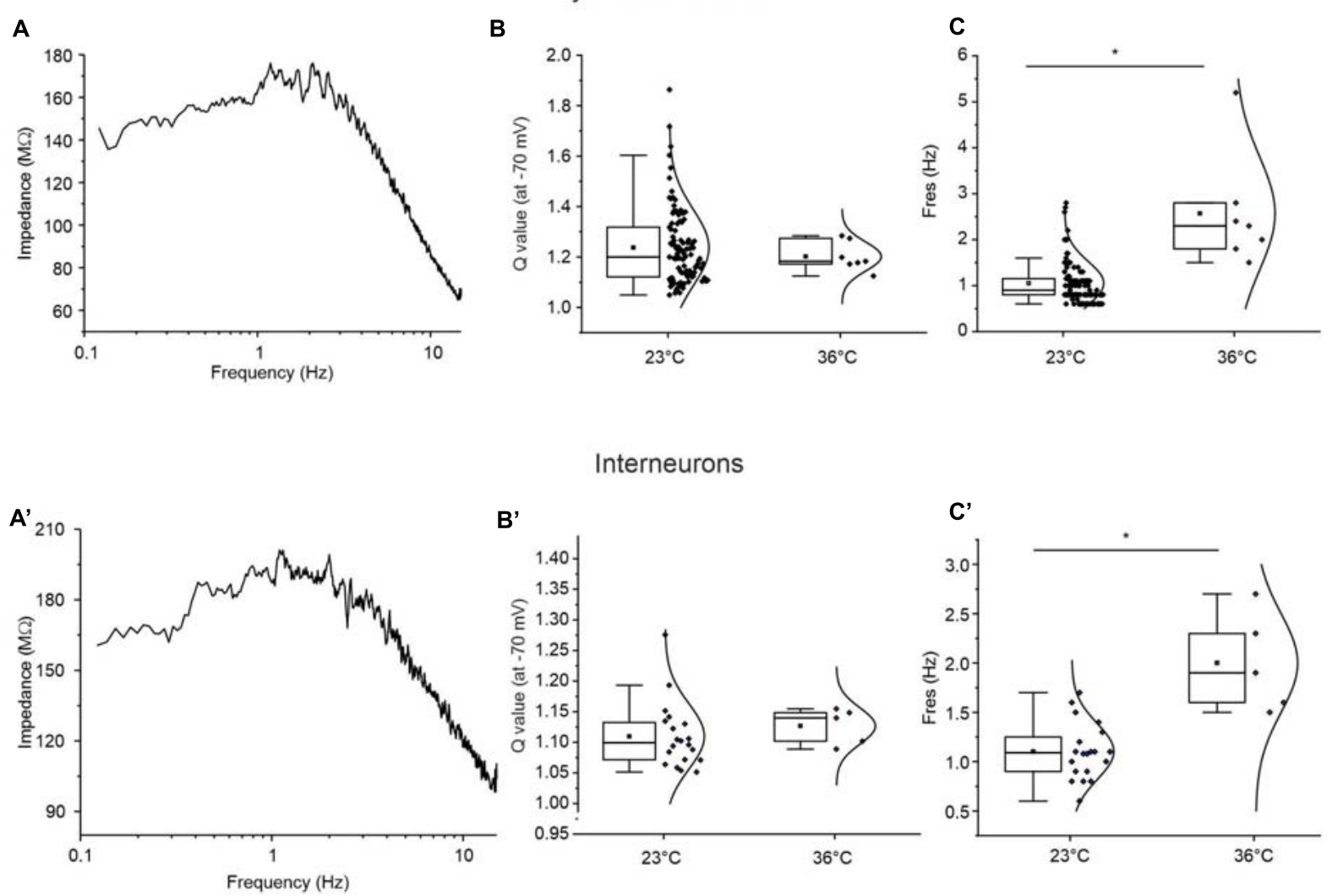

FIGURE 10 | Effect of temperature on the resonance properties of pyramidal neurons $(\mathbf{A}-\mathbf{C})$ and $G A B A e r g i c$ interneurons $\left(\mathbf{A}^{\prime}-\mathbf{C}^{\prime}\right)$ of the PRC.

(A) Impedance-to-frequency relationship obtained from a representative resonant pyramidal neuron at a temperature of $36^{\circ} \mathrm{C}$ by applying the standard $0-15 \mathrm{~Hz} Z \mathrm{AP}$ protocol at $-70 \mathrm{mV}$. (B,C) All-points half-violin plots flanked by summary box plots comparing the $Q$-values $(\mathbf{B})$ and $F_{\text {res }}$ values $(\mathbf{C})$ derived from resonant pyramidal neurons at room temperature $\left(23^{\circ} \mathrm{C}\right)$ and at physiological temperature $\left(36^{\circ} \mathrm{C}\right)$. The increase of temperature in the 7 pyramidal neurons tested at $36^{\circ} \mathrm{C}$ has no influence on the $Q$-value $\mathbf{( B )}$, while determines a significant increase of $F_{\text {res }}(\mathbf{C})$. ( $\left.\mathbf{A}^{\prime}-\mathbf{C}^{\prime}\right)$ Same as $(\mathbf{A}-\mathbf{C})$ for resonant $G A B A e r g i c$ interneurons $(N=5)$. Note that also in this cell type, the temperature increase does not alter the $Q$-value $\left(\mathbf{B}^{\prime}\right)$, while determines a significant increase of the $F_{\text {res }}\left(\mathbf{C}^{\prime}\right)$. Data in $\left(\mathbf{B}, \mathbf{C}, \mathbf{B}^{\prime} \mathbf{C}^{\prime}\right)$ were compared by unpaired Student's $t$-test. ${ }^{*} p<0.05$.

electrophysiological parameters are quite conserved (Zhou and Lipsius, 1992; Kiss, 2008; Kase and Imoto, 2012), we believe that reliable results could be obtained also by using general equations. Indeed, our computational model faithfully reproduced the experimental behavior, confirming the indispensable role of $\mathrm{I}_{\mathrm{h}}$ in the genesis of resonance and the involvement of the persistent sodium current in the amplification of the resonance peak. Furthermore, it is important to consider that temperature strongly influences the $\mathrm{F}_{\text {res }}$ and this happens also in PRC, as predicted by our computational model and confirmed by our experimental data. We found that the rise of temperature from 23 to $36^{\circ} \mathrm{C}$ significantly increased the $\mathrm{F}_{\text {res }}$ of both pyramidal neurons and interneurons, aligning these data to the wellknown resonance frequency already observed in the brain areas functionally linked to the PRC.

The functional behavior of the individual neurons affects and defines the processing of information within neural circuits. Membrane resonance at a given frequency has already been described in many other cells across different brain areas and is strictly dependent on intrinsic membrane properties, synaptic inputs, and modulatory effects. The interaction between the passive properties of the cell and active conductances mediated by ion channels define not only membrane resonance but also action potential timing, synaptic integration, and membrane potential oscillations. Altogether these properties enable neurons to react preferentially to synaptic inputs at specific frequencies and also influence the dynamics of the neural networks of which they are part. The inhibition produced by interneurons on pyramidal neurons and other interneurons is critical for the synchronization of neural activity (Mann and Paulsen, 2007). In the PRC the resonance of interneurons at $1-3 \mathrm{~Hz}$ suggests that synaptic inputs at these frequencies will likely entrain the entire neuronal network of the PRC to oscillate at the range of 1-3 Hz, typical of the delta rhythm during non REM sleep.

Coherent network oscillations distributed in different brain regions are critical to achieve learning and memory and in general to process neural information. Low frequency oscillations are usually engaged to connect different cerebral regions to transfer or retrieve distributed information. On the other hand, coherent high frequency oscillations are more restricted to 


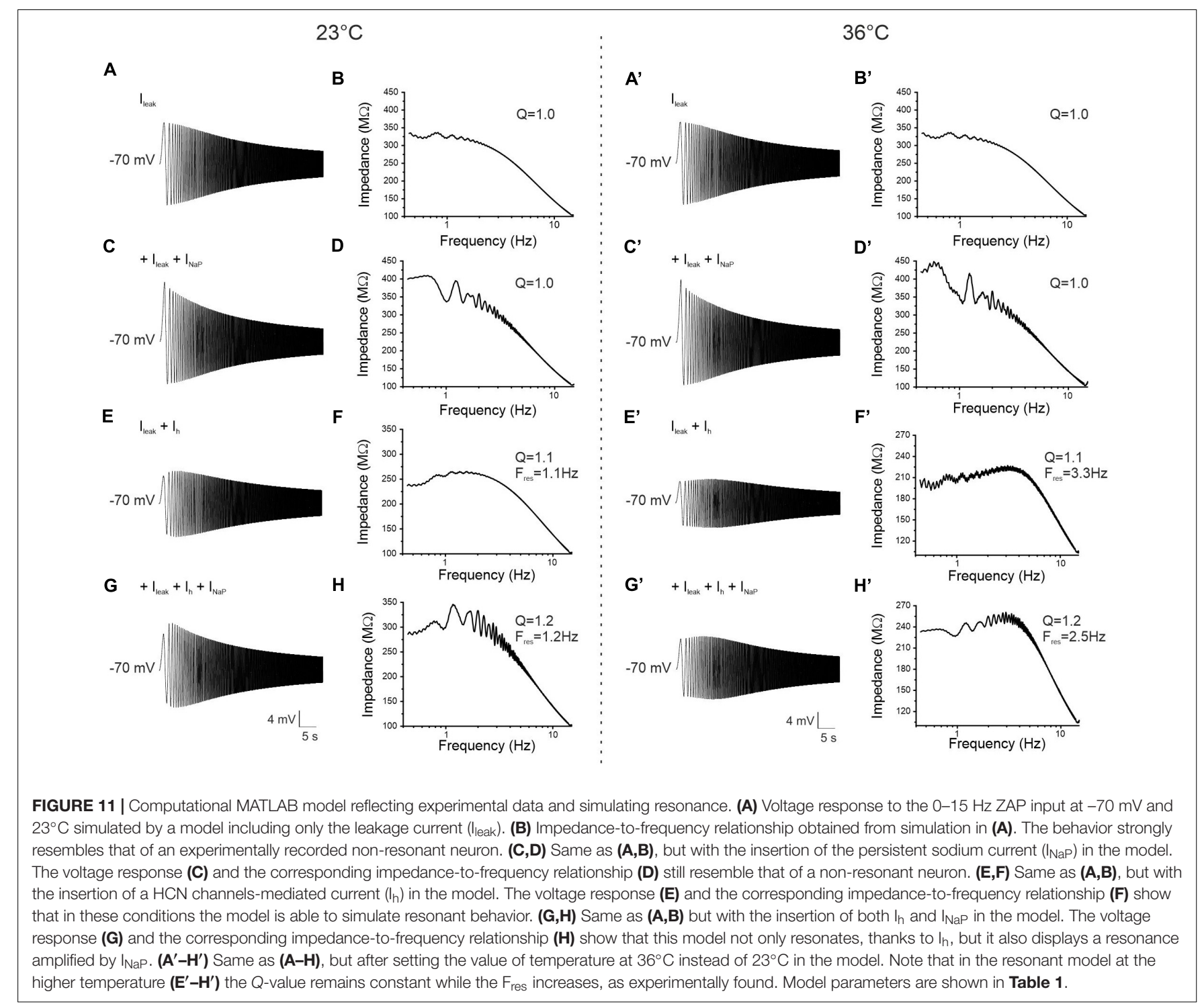

localized neuronal networks and seem to be involved in encoding of sensory information, in promoting synaptic plasticity (Fries, 2005), and in the definition of the sequence of information transfer (Sejnowski and Paulsen, 2006; Fries et al., 2007).

Semantic memories are stored in different cortical areas and a still unanswered question is how these distributed memories are recalled and linked together to obtain an object representation. In the PRC, the representation of the objects learned throughout experience-dependent episodes are associated with their memorized features. The retrieving of these associations is based on the information exchange among the PRC and distributed cortical areas. The PRC, hence, via its afferent and efferent connections acts as a hub of semantic memory.

Slow frequency oscillations $(0.5-4 \mathrm{~Hz})$ that are widely described in different areas linked to PRC such as the neocortex, amygdala, and lateral EC (Collins et al., 2001) could subserve the pivotal role, played by the PRC, for binding the information stored sparsely in different cortical areas to obtain a coherent neural representation to be sent to the hippocampus. Similarly, but in opposite direction, short-term memory developed in hippocampus should be forwarded and stored in distributed neocortical regions throughout a consolidation process that likely use the same PRC hub and oscillatory mechanisms. The reverberating activity between neocortex and hippocampus, especially during slow wave sleep, indeed represents the mechanism by which the brain promotes the redistribution and strengthening of memory representation in cortical regions (Klinzing et al., 2019). Another aspect which worth to be considered is related with dendritic resonance. Detailed studies have characterized the spatial properties of the resonance along the dendrite-to-soma extension in hippocampal (Narayanan and Johnston, 2007; Hu et al., 2009) and prefrontal (Kalmbach et al., 2013) pyramidal neurons. In particular, recordings performed on CA1 pyramidal neurons showed that membrane resonance frequency could differ threefold between soma and apical 
dendrites. These results suggest that CA1 pyramidal cells could act as stimulus-tuned filters for spatially separated synaptic inputs. The cognitive processes of the PRC strongly rely on the presence of separated synaptic inputs from piriform, entorhinal and neocortical cortices, and amygdala to the PRC and its ability to integrate them. Then, future experiments should also be addressed to investigate the resonance properties along the soma-to-dendrite extension in pyramidal neurons of the PRC.

\section{DATA AVAILABILITY STATEMENT}

The raw data supporting the conclusions of this article will be made available by the authors, without undue reservation.

\section{ETHICS STATEMENT}

The animal study was reviewed and approved by the National Ministry of Health and Local Ethical Committee of the University of Pavia (OPBA).

\section{REFERENCES}

Aracri, P., Colombo, E., Mantegazza, M., Scalmani, P., Curia, G., Avanzini, G., et al. (2006). Layer-specific properties of the persistent sodium current in sensorimotor cortex. J. Neurophysiol. 95, 3460-3468. doi: 10.1152/jn.00588. 2005

Beggs, J. M., Moyer, J. R., McGann, J. P., and Brown, T. H. (2000). Prolonged synaptic integration in perirhinal cortical neurons. J. Neurophysiol. 83, 32943298. doi: 10.1152/jn.2000.83.6.3294

Biel, M., Wahl-Schott, C., Michalakis, S., and Zong, X. (2009). Hyperpolarizationactivated cation channels. from genes to function. Physiol. Rev. 89, 847-885. doi: 10.1152/physrev.00029.2008

Biella, G., Spaiardi, P., Toselli, M., de Curtis, M., and Gnatkovsky, V. (2010). Functional interactions within the parahippocampal region revealed by voltagesensitive dye imaging in the isolated guinea pig brain. J. Neurophysiol. 103, 725-732. doi: 10.1152/jn.00722.2009

Biella, G., Uva, L., and de Curtis, M. (2001). Network activity evoked by neocortical stimulation in area 36 of the guinea pig perirhinal cortex. J. Neurophysiol. 86, 164-172. doi: 10.1152/jn.2001.86.1.164

Biella, G., Uva, L., and de Curtis, M. (2002). Propagation of neuronal activity along the neocortical-perirhinal-entorhinal pathway in the guinea pig. J. Neurosci. 22, 9972-9979. doi: 10.1523/jneurosci.22-22-09972.2002

Boehlen, A., Henneberger, C., Heinemann, U., and Erchova, I. (2013). Contribution of near-threshold currents to intrinsic oscillatory activity in rat medial entorhinal cortex layer II stellate cells. J. Neurophysiol. 109, 445-463. doi: 10.1152/jn.00743.2011

Burke, S. N., Gaynor, L. S., Barnes, C. A., Bauer, R. M., Bizon, J. L., Roberson, E. D., et al. (2018). Shared functions of perirhinal and parahippocampal cortices: implications for cognitive aging. Trends Neurosci. 41, 349-359. doi: 10.1016/j. tins.2018.03.001

Chen, W. R., and Shepherd, G. M. (1997). Membrane and synaptic properties of mitral cells in slices of rat olfactory bulb. Brain Res. 745, 189-196. doi: 10.1016/s0006-8993(96)01150-x

Collins, D. R., Pelletier, J. G., and Paré, D. (2001). Slow and fast (gamma) neuronal oscillations in the perirhinal cortex and lateral amygdala. J. Neurophysiol. 85, 1661-1672. doi: 10.1152/jn.2001.85.4.1661

Davachi, L. (2006). Item, context and relational episodic encoding in humans. Curr. Opin. Neurobiol. 16, 693-700. doi: 10.1016/j.conb.2006.10.012

de Curtis, M., and Paré, D. (2004). The rhinal cortices: a wall of inhibition between the neocortex and the hippocampus. Prog Neurobiol 74, 101-110. doi: 10.1016/ j.pneurobio.2004.08.005

\section{AUTHOR CONTRIBUTIONS}

NB, FT, PS, and GB conceived the study and designed the experiments. NB, FT, PS, CM, FR, NM, and AC performed the in vitro experiments and data analysis. MP, SR, and MT defined and performed the computational model. YY developed and provided the GAD67-GFP mice. CD edited the manuscript. NB, FT, MT, CM, and GB wrote the manuscript. GB supervised the study. All authors contributed to the article and approved the submitted version.

\section{FUNDING}

This work was supported by the Italian Ministry of Education, University and Research (MIUR): 2005059453_003, Dipartimenti di Eccellenza Program (2018-2022)-Department of Biology and Biotechnology "Lazzaro Spallanzani," University of Pavia and Crowdfunding Campaign "La Cura in una Cellula"University of Pavia. FT was supported by the Fondazione Umberto Veronesi.

Desmaisons, D., Vincent, J. D., and Lledo, P. M. (1999). Control of action potential timing by intrinsic subthreshold oscillations in olfactory bulb output neurons. J. Neurosci. 19, 10727-10737. doi: 10.1523/jneurosci.19-24-10727.1999

Ehrlich, D. E., Ryan, S. J., and Rainnie, D. G. (2012). Postnatal development of electrophysiological properties of principal neurons in the rat basolateral amygdala. J. Physiol. 590, 4819-4838. doi: 10.1113/jphysiol.2012.237453

Engel, T. A., Schimansky-Geier, L., Herz, A. V., Schreiber, S., and Erchova, I. (2008). Subthreshold membrane-potential resonances shape spike-train patterns in the entorhinal cortex. J. Neurophysiol. 100, 1576-1589. doi: 10.1152/jn.01282. 2007

Erchova, I., Kreck, G., Heinemann, U., and Herz, A. V. (2004). Dynamics of rat entorhinal cortex layer II and III cells: characteristics of membrane potential resonance at rest predict oscillation properties near threshold. J. Physiol. 560, 89-110. doi: 10.1113/jphysiol.2004.069930

Faulkner, B., and Brown, T. H. (1999). Morphology and physiology of neurons in the rat perirhinal-lateral amygdala area. J. Comp. Neurol. 411, 613-642.

Fries, P. (2005). A mechanism for cognitive dynamics. neuronal communication through neuronal coherence. Trends Cogn. Sci. 9, 474-480. doi: 10.1016/j.tics. 2005.08.011

Fries, P., Nikolić, D., and Singer, W. (2007). The gamma cycle. Trends Neurosci. 30, 309-316.

Garden, D. L., Kemp, N., and Bashir, Z. I. (2002). Differences in GABAergic transmission between two inputs into the perirhinal cortex. Eur. J. Neurosci. 16, 437-444. doi: 10.1046/j.1460-9568.2002.02096.x

Graf, M., Nair, A., Wong, K. L. L., Tang, Y., and Augustine, G. J. (2020). Identification of mouse claustral neuron types based on their intrinsic electrical properties. eNeuro 7:ENEURO.0216-20.2020.

Gutfreund, Y., yarom, Y., and Segev, I. (1995). Subthreshold oscillations and resonant frequency in guinea-pig cortical neurons. physiology and modelling. $J$ Physiol 483(Pt 3), 621-640. doi: 10.1113/jphysiol.1995.sp020611

Haas, J. S., and White, J. A. (2002). Frequency selectivity of layer II stellate cells in the medial entorhinal cortex. J. Neurophysiol. 88, 2422-2429. doi: 10.1152/jn. 00598.2002

Hedrich, U. B., Liautard, C., Kirschenbaum, D., Pofahl, M., Lavigne, J., Liu, Y., et al. (2014). Impaired action potential initiation in GABAergic interneurons causes hyperexcitable networks in an epileptic mouse model carrying a human $\mathrm{Na}(\mathrm{V}) 1.1$ mutation. J. Neurosci. 34, 14874-14889. doi: 10.1523/jneurosci.072114.2014

Hodgkin, A. L., and Huxley, A. F. (1952). A quantitative description of membrane current and its application to conduction and excitation in nerve. J. Physiol. 117, 500-544. doi: 10.1113/jphysiol.1952.sp004764 
Holt, G. R., Softky, W. R., Koch, C., and Douglas, R. J. (1996). Comparison of discharge variability in vitro and in vivo in cat visual cortex neurons. J. Neurophysiol. 75, 1806-1814. doi: 10.1152/jn.1996.75. 5.1806

Hu, H., Vervaeke, K., Graham, L. J., and Storm, J. F. (2009). Complementary theta resonance filtering by two spatially segregated mechanisms in CA1 hippocampal pyramidal neurons. J. Neurosci. 29, 14472-14483. doi: 10.1523/ jneurosci.0187-09.2009

Hu, H., Vervaeke, K., and Storm, J. F. (2002). Two forms of electrical resonance at theta frequencies, generated by $\mathrm{M}$-current, $\mathrm{h}$-current and persistent $\mathrm{Na}+$ current in rat hippocampal pyramidal cells. J. Physiol. 545, 783-805. doi: 10.1113/jphysiol.2002.029249

Hutcheon, B., Miura, R. M., and Puil, E. (1996). Subthreshold membrane resonance in neocortical neurons. J. Neurophysiol. 76, 683-697. doi: 10.1152/jn.1996.76.2. 683

Hutcheon, B., and Yarom, Y. (2000). Resonance, oscillation and the intrinsic frequency preferences of neurons. Trends Neurosci. 23, 216-222. doi: 10.1016/ s0166-2236(00)01547-2

Izhikevich, E. M., Desai, N. S., Walcott, E. C., and Hoppensteadt, F. C. (2003). Bursts as a unit of neural information: selective communication via resonance. Trends Neurosci. 26, 161-167.

Johnson, S. W., and Seutin, V. (1997). Bicuculline methiodide potentiates NMDAdependent burst firing in rat dopamine neurons by blocking apamin-sensitive Ca2+-activated K+ currents. Neurosci. Lett. 231, 13-16. doi: 10.1016/s03043940(97)00508-9

Kalmbach, B. E., Chitwood, R. A., Dembrow, N. C., and Johnston, D. (2013). Dendritic generation of mGluR-mediated slow afterdepolarization in layer 5 neurons of prefrontal cortex. J. Neurosci. 33, 13518-13532. doi: 10.1523/ jneurosci.2018-13.2013

Karayannis, T., Huerta-Ocampo, I., and Capogna, M. (2007). GABAergic and pyramidal neurons of deep cortical layers directly receive and differently integrate callosal input. Cereb. Cortex 17, 1213-1226. doi: 10.1093/cercor/ bhl035

Kase, D., and Imoto, K. (2012). The role of HCN channels on membrane excitability in the nervous system. J. Signal Transduct. 2012:619747.

Kealy, J., and Commins, S. (2011). The rat perirhinal cortex: a review of anatomy, physiology, plasticity, and function. Prog. Neurobiol. 93, 522-548. doi: 10.1016/ j.pneurobio.2011.03.002

Kiss, T. (2008). Persistent Na-channels: origin and function. A review. Acta Biol. Hung. 59(Suppl.), 1-12. doi: 10.1556/abiol.59.2008.suppl.1

Klinzing, J. G., Niethard, N., and Born, J. (2019). Mechanisms of systems memory consolidation during sleep. Nat. Neurosci. 22, 1598-1610. doi: 10.1038/s41593019-0467-3

Komendantov, A. O., Venkadesh, S., Rees, C. L., Wheeler, D. W., Hamilton, D. J., and Ascoli, G. A. (2019). Quantitative firing pattern phenotyping of hippocampal neuron types. Sci. Rep. 9:17915.

Lampl, I., and Yarom, Y. (1997). Subthreshold oscillations and resonant behavior: two manifestations of the same mechanism. Neuroscience 78, 325-341. doi: 10.1016/s0306-4522(96)00588-x

Leung, L. S., and Yu, H. W. (1998). Theta-frequency resonance in hippocampal CA1 neurons in vitro demonstrated by sinusoidal current injection. J. Neurophysiol. 79, 1592-1596. doi: 10.1152/jn.1998.79.3.1592

Ma, Y., Hu, H., Berrebi, A. S., Mathers, P. H., and Agmon, A. (2006). Distinct subtypes of somatostatin-containing neocortical interneurons revealed in transgenic mice. J. Neurosci. 26, 5069-5082. doi: 10.1523/jneurosci.0661-06. 2006

Magee, J. C. (1998). Dendritic hyperpolarization-activated currents modify the integrative properties of hippocampal CA1 pyramidal neurons. J. Neurosci. 18, 7613-7624. doi: 10.1523/jneurosci.18-19-07613.1998

Mann, E. O., and Paulsen, O. (2007). Role of GABAergic inhibition in hippocampal network oscillations. Trends Neurosci. 30, 343-349. doi: 10.1016/j.tins.2007. 05.003

Marder, E., and Taylor, A. L. (2011). Multiple models to capture the variability in biological neurons and networks. Nat. Neurosci. 14, 133-138. doi: 10.1038/nn. 2735

Martina, M., Royer, S., and Paré, D. (2001). Propagation of neocortical inputs in the perirhinal cortex. J. Neurosci. 21, 2878-2888. doi: 10.1523/jneurosci.21-0802878.2001
Matsumoto-Makidono, Y., Nakayama, H., Yamasaki, M., Miyazaki, T., Kobayashi, K., Watanabe, M., et al. (2016). Ionic basis for membrane potential resonance in neurons of the inferior olive. Cell Rep. 16:994-1004. doi: 10.1016/j.celrep.2016. 06.053

McGann, J. P., Moyer, J. R., and Brown, T. H. (2001). Predominance of latespiking neurons in layer VI of rat perirhinal cortex. J. Neurosci. 21, 4969-4976. doi: 10.1523/jneurosci.21-14-04969.2001

Mishra, P., and Narayanan, R. (2020). Heterogeneities in intrinsic excitability and frequency-dependent response properties of granule cells across the blades of the rat dentate gyrus. J. Neurophysiol. 123, 755-772. doi: 10.1152/jn.00443. 2019

Murray, E. A., and Richmond, B. J. (2001). Role of perirhinal cortex in object perception, memory, and associations. Curr. Opin. Neurobiol. 11, 188-193. doi: 10.1016/s0959-4388(00)00195-1

Narayanan, R., and Johnston, D. (2007). Long-term potentiation in rat hippocampal neurons is accompanied by spatially widespread changes in intrinsic oscillatory dynamics and excitability. Neuron 56, 1061-1075.

Nolan, M. F., Dudman, J. T., Dodson, P. D., and Santoro, B. (2007). HCN1 channels control resting and active integrative properties of stellate cells from layer II of the entorhinal cortex. J. Neurosci. 27, 12440-12451.

Pedroarena, C. M., Pose, I. E., Yamuy, J., Chase, M. H., and Morales, F. R. (1999). Oscillatory membrane potential activity in the soma of a primary afferent neuron. J. Neurophysiol. 82, 1465-1476. doi: 10.1152/jn.1999.82.3.1465

Perugini, A., Laing, M., Berretta, N., Aicardi, G., and Bashir, Z. I. (2012). Synaptic plasticity from amygdala to perirhinal cortex: a possible mechanism for emotional enhancement of visual recognition memory? Eur. J. Neurosci. 36, 2421-2427. doi: 10.1111/j.1460-9568.2012.08146.x

Pike, F. G., Goddard, R. S., Suckling, J. M., Ganter, P., Kasthuri, N., and Paulsen, O. (2000). Distinct frequency preferences of different types of rat hippocampal neurones in response to oscillatory input currents. J. Physiol. 529(Pt 1), 205213. doi: $10.1111 /$ j.1469-7793.2000.00205.x

Pinto, A., Fuentes, C., and Paré, D. (2006). Feedforward inhibition regulates perirhinal transmission of neocortical inputs to the entorhinal cortex: ultrastructural study in guinea pigs. J. Comp. Neurol. 495, 722-734. doi: 10 . 1002/cne.20905

Rathour, R. K., and Narayanan, R. (2019). Degeneracy in hippocampal physiology and plasticity. Hippocampus 29, 980-1022. doi: 10.1002/hipo.23139

Schaefer, A. T., Angelo, K., Spors, H., and Margrie, T. W. (2006). Neuronal oscillations enhance stimulus discrimination by ensuring action potential precision. PLoS Biol. 4:e163. doi: 10.1371/journal.pbio.0040163

Sejnowski, T. J., and Paulsen, O. (2006). Network oscillations. emerging computational principles. J Neurosci 26, 1673-1676. doi: 10.1523/jneurosci. 3737-05d.2006

Shay, C. F., Boardman, I. S., James, N. M., and Hasselmo, M. E. (2012). Voltage dependence of subthreshold resonance frequency in layer II of medial entorhinal cortex. Hippocampus 22, 1733-1749. doi: 10.1002/hipo.22008

Shinomoto, S., Kim, H., Shimokawa, T., Matsuno, N., Funahashi, S., Shima, K., et al. (2009). Relating neuronal firing patterns to functional differentiation of cerebral cortex. PLoS Comput. Biol. 5:e1000433. doi: 10.1371/journal.pcbi.1000433

Spain, W. J., Schwindt, P. C., and Crill, W. E. (1987). Anomalous rectification in neurons from cat sensorimotor cortex in vitro. J. Neurophysiol. 57, 1555-1576. doi: 10.1152/jn.1987.57.5.1555

Staresina, B. P., and Davachi, L. (2008). Selective and shared contributions of the hippocampus and perirhinal cortex to episodic item and associative encoding. J. Cogn. Neurosci. 20, 1478-1489. doi: 10.1162/jocn.2008.20104

Sun, H., An, S., Luhmann, H. J., and Kilb, W. (2014). Resonance properties of GABAergic interneurons in immature GAD67-GFP mouse neocortex. Brain Res 1548, 1-11. doi: 10.1016/j.brainres.2013.12.032

Suzuki, W. A., and Naya, Y. (2014). The perirhinal cortex. Annu Rev Neurosci 37, $39-53$.

Tamamaki, N., Yanagawa, Y., Tomioka, R., Miyazaki, J., Obata, K., and Kaneko, T. (2003). Green fluorescent protein expression and colocalization with calretinin, parvalbumin, and somatostatin in the GAD67-GFP knock-in mouse. J. Comp. Neurol. 467, 60-79. doi: 10.1002/cne.10905

Traub, R. D., and Miles, R. (1991). Multiple modes of neuronal population activity emerge after modifying specific synapses in a model of the CA3 region of the hippocampus. Ann. N. Y. Acad. Sci. 627, 277-290. doi: 10.1111/j.1749-6632. 1991.tb25931.x 
Vera, J., Pezzoli, M., Pereira, U., Bacigalupo, J., and Sanhueza, M. (2014). Electrical resonance in the $\theta$ frequency range in olfactory amygdala neurons. PLoS One 9:e85826. doi: 10.1371/journal.pone.0085826

Willems, J. G. P., Wadman, W. J., and Cappaert, N. L. M. (2018). Parvalbumin interneuron mediated feedforward inhibition controls signal output in the deep layers of the perirhinal-entorhinal cortex. Hippocampus 28, 281-296. doi: 10. 1002/hipo.22830

Witter, M. P., Wouterlood, F. G., Naber, P. A., and Van Haeften, T. (2000). Anatomical organization of the parahippocampal-hippocampal network. Ann. N. Y. Acad. Sci. 911, 1-24. doi: 10.1111/j.1749-6632.2000.tb06716.x

Wu, N., Hsiao, C. F., and Chandler, S. H. (2001). Membrane resonance and subthreshold membrane oscillations in mesencephalic $\mathrm{V}$ neurons: participants in burst generation. J. Neurosci. 21, 3729-3739. doi: 10.1523/jneurosci.21-1103729.2001

Xue, W. N., Wang, Y., He, S. M., Wang, X. L., Zhu, J. L., and Gao, G. D. (2012). SK- and h-current contribute to the generation of theta-like resonance of rat substantia nigra pars compacta dopaminergic neurons at hyperpolarized membrane potentials. Brain Struct. Funct. 217, 379-394. doi: 10.1007/s00429011-0361-6
Yan, Z. Q., Liu, S. M., Li, J., Wang, Y., Gao, L., Xie, R. G., et al. (2012). Membrane resonance and its ionic mechanisms in rat subthalamic nucleus neurons. Neurosci. Lett. 506, 160-165. doi: 10.1016/j.neulet.2011.10.072

Zhou, Z., and Lipsius, S. L. (1992). Properties of the pacemaker current (If) in latent pacemaker cells isolated from cat right atrium. J. Physiol. 453, 503-523. doi: 10.1113/jphysiol.1992.sp019242

Conflict of Interest: The authors declare that the research was conducted in the absence of any commercial or financial relationships that could be construed as a potential conflict of interest.

Copyright (๔ 2021 Binini, Talpo, Spaiardi, Maniezzi, Pedrazzoli, Raffin, Mattiello, Castagno, Masetto, Yanagawa, Dickson, Ramat, Toselli and Biella. This is an openaccess article distributed under the terms of the Creative Commons Attribution License (CC BY). The use, distribution or reproduction in other forums is permitted, provided the original author(s) and the copyright owner(s) are credited and that the original publication in this journal is cited, in accordance with accepted academic practice. No use, distribution or reproduction is permitted which does not comply with these terms. 